\title{
Afterglow light curves from misaligned structured jets
}

\author{
Paz Beniamini ${ }^{\oplus},{ }^{1 \star}$ Jonathan Granot ${ }^{2,3}$ and Ramandeep Gill ${ }^{2,3}$ \\ ${ }^{1}$ Division of Physics, Mathematics and Astronomy, California Institute of Technology, Pasadena, CA 91125, USA \\ ${ }^{2}$ Department of Natural Sciences, The Open University of Israel, 1 University Road, P.O. Box 808, Raanana 4353701, Israel \\ ${ }^{3}$ Department of Physics, The George Washington University, Washington, DC 20052, USA
}

Accepted 2020 February 21. Received 2020 February 21; in original form 2020 January 7

\begin{abstract}
GRB 170817A/GW 170817 is the first gamma-ray burst (GRB) clearly viewed far from the GRB jet's symmetry axis. Its afterglow was densely monitored over a wide range of frequencies and times. It has been modelled extensively, primarily numerically, and although this endeavour was very fruitful, many of the underlying model parameters remain undetermined. We provide analytic modelling of GRB afterglows observed off-axis, considering jets with a narrow core (of half-opening angle $\left.\theta_{\mathrm{c}}\right)$ and power-law wings in energy per unit solid angle $\left(\epsilon=\epsilon_{\mathrm{c}} \Theta^{-a}\right.$ where $\left.\Theta=\left[1+\left(\theta / \theta_{c}\right)^{2}\right]^{1 / 2}\right)$ and initial specific kinetic energy $\left(\Gamma_{0}-1=\left[\Gamma_{\mathrm{c}, 0}-1\right] \Theta^{-b}\right)$, as well as briefly discuss Gaussian jets. Our study reveals qualitatively different types of light curves that can be viewed in future off-axis GRBs, with either single or double peaks, depending on the jet structure and the viewing angle. Considering the light-curve shape rather than the absolute normalizations of times and/or fluxes, removes the dependence of the light curve on many of the highly degenerate burst parameters. This study can be easily used to determine the underlying jet structure, significantly reduce the effective parameter space for numerical fitting attempts and provide physical insights. As an illustration, we show that for GRB 170817A, there is a strong correlation between the allowed values of $\Gamma_{\mathrm{c}, 0}$ and $b$, leading to a narrow strip of allowed solutions in the $\Gamma_{\mathrm{c}, 0}-b$ plane above some minimal values $\Gamma_{\mathrm{c}, 0} \gtrsim$ $40, b \gtrsim 1.2$. Furthermore, the Lorentz factor of the material dominating the early light curve can be constrained by three independent techniques to be $\Gamma_{0}\left(\theta_{\min , 0}\right) \approx 5-7$.
\end{abstract}

Key words: radiation mechanisms: general-gamma-ray burst: general.

\section{INTRODUCTION}

The detection of a binary neutron star merger with laser interferometer gravitational-wave observatory (LIGO), GW170817 (Abbott et al. 2017a), accompanied by a long-lived gamma-ray burst (GRB) afterglow (e.g. Abbott et al. 2017b) has enabled us for the first time to unambiguously observe the afterglow of a GRB seen from latitudes much greater than the jet's core. The late peak and the slow rise of the light curve towards that peak have been modelled as arising due to either the angular (Granot, Guetta \& Gill 2017; Lamb \& Kobayashi 2017; Lazzati et al. 2017; Gill \& Granot 2018; Kathirgamaraju, Barniol Duran \& Giannios 2018) or radial (Kasliwal et al. 2017; Gill \& Granot 2018; Gottlieb et al. 2018; Nakar \& Piran 2018) structure of the outflow. The observation of superluminal motion (Mooley et al. 2018; Ghirlanda et al. 2019), as well as the sharp decline of the light curve after the peak (e.g. D'Avanzo et al. 2018; Lamb, Mandel \& Resmi 2018; Margutti et al. 2018; Troja et al. 2018; Hajela et al. 2019; Lamb et al. 2019) suggests the flow had an energetic and relativistic compact core rather than a

^E-mail: paz.beniamini@gmail.com quasi-spherical structure, implying that an angular structured jet is required to explain, at least the late time afterglow observations.

Determining the angular structure of GRB jets outside of their cores is of crucial importance for advancing our knowledge of various phenomena, such as the physics of formation and dynamics of relativistic jets (Granot, De Colle \& Ramirez-Ruiz 2018; Kathirgamaraju et al. 2018; Beniamini et al. 2020a), the underlying mechanism powering the prompt phase of GRBs (Beniamini et al. 2019; Beniamini \& Nakar 2019), and possibly some phenomena observed in cosmological GRB afterglows, like X-ray plateaus (Eichler \& Granot 2006; Beniamini et al. 2020b; Oganesyan et al. 2019).

Numerical modelling of off-axis GRB jets with an angular structure has been extensively studied in the literature in order to try and determine the underlying physical properties of the burst and the surrounding medium (e.g. Alexander et al. 2018; Lamb \& Kobayashi 2018; Lazzati et al. 2018; Xie, Zrake \& MacFadyen 2018; Ryan et al. 2019). Although such modelling is extremely useful it encounters two significant limitations. The first is the large parameter space that must be explored in order to determine those properties. This results in significant computational costs involved in thorough modelling attempts. The second limitation 
is due to the degeneracies between the intrinsic parameters in their effects on the final light curves. Both of these limitations can be largely overcome with the aid of analytical modelling. The latter can reduce the effective parameter space and point towards the unknown parameters (or combinations of those) that may be well constrained by observations (e.g. Gill et al. 2019).

Analytical modelling of GRB afterglows arising from a jet with angular structure have been carried out by various authors (e.g. Rossi, Lazzati \& Rees 2002; Granot \& Kumar 2003; Kumar \& Granot 2003; Panaitescu \& Kumar 2003; Rossi et al. 2004; Granot, Ramirez-Ruiz \& Perna 2005; Eichler \& Granot 2006). Here, motivated by GRB 170817A, and the expectation of seeing future similarly off-axis GRBs triggered by gravitational waves (GW) detections, we systematically consider the afterglow light curves for GRBs that are viewed far from the jet axis. Analytical treatment of this situation reveals qualitatively different types of evolution that may be seen by different observers. In particular, we find that either single- or double-peaked light curves can be obtained and the distinction between the two is directly related to the jet structure and the observer's viewing angle.

The paper is organized as follows. In Section 2, we introduce the basic definitions of the jet properties including its angular structure, and describe the dynamics underlying the GRB afterglow as viewed in observer frame coordinates. In Section 3, we describe the contributions from different regions (or latitudes) in the jet to the observed emission and their temporal evolution. In Section 4, we examine the different types of afterglow light curves that can be viewed depending on the jet properties and the observation angle. We present in Section 5 the relation between the observable properties of off-axis GRB afterglows and the underlying physical properties and show which of the latter can be robustly determined by the former. In Section 6, we demonstrate the effectiveness of this technique on GRB 170817A. We finally conclude in Section 7.

\section{POWER-LAW STRUCTURED JET: DYNAMICS AND SCALINGS}

For simplicity, we consider a jet angular structure consisting of a narrow core of half-opening angle $\theta_{c}$ that smoothly transitions into power-law (PL) wings in both the kinetic energy per unit solid angle, $\epsilon \equiv \mathrm{d} E_{\mathrm{k}} / d \Omega$, and the initial specific kinetic energy, $\Gamma_{0}-1$. For concreteness, we will mainly focus in this paper on PL profiles for both quantities (following Granot \& Kumar 2003) but will briefly discuss implications for Gaussian jets in Section 4.4. In the former case, the energy and Lorentz factor are given by

$$
\frac{\epsilon(\theta)}{\epsilon_{\mathrm{c}}}=\Theta^{-a}, \quad \frac{\Gamma_{0}(\theta)-1}{\Gamma_{\mathrm{c}, 0}-1}=\Theta^{-b}, \quad \Theta \equiv \sqrt{1+\left(\frac{\theta}{\theta_{\mathrm{c}}}\right)^{2}},
$$

where $\theta$ is the polar angle measured from the jet's symmetry axis ( $\theta_{\mathrm{obs}}$ is the polar angle of the observer's line of sight, LOS). We also define $\xi_{\mathrm{c}} \equiv\left(\Gamma_{\mathrm{c}, 0} \theta_{\mathrm{c}}\right)^{2}$ and $q \equiv \theta_{\mathrm{obs}} / \theta_{\mathrm{c}}$ such that $\Theta_{\mathrm{obs}}=\sqrt{1+q^{2}}$, which will be useful quantities later on. We consider emission form a thin shell behind the external shock that is radially located at $R(\theta$, $t$ ), where each point on the jet expands as if part of a spherical flow with no lateral expansion.

To a first approximation, and as long as the flow is still ultrarelativistic, $\Gamma(\theta)$ may be assumed to be constant up to the deceleration radius $R_{\operatorname{dec}}(\theta)$, after which it starts decelerating as a PL in radius,

$\Gamma(\theta, R)=\Gamma_{0}(\theta) \times \begin{cases}1 & R<R_{\mathrm{dec}}(\theta) \\ \zeta^{\frac{k-3}{2}} & R>R_{\mathrm{dec}}(\theta),\end{cases}$ where $\zeta \equiv R / R_{\mathrm{dec}}(\theta), k$ relates the external density to the radius, i.e. $\rho=A R^{-k}$ and where the values of the deceleration radii, $R_{\operatorname{dec}}(\theta)$, can conveniently be scaled compared to that at the core

$R_{\mathrm{dec}, \mathrm{c}}=\left[\frac{(3-k) \epsilon_{c}}{A c^{2} \Gamma_{\mathrm{c}, 0}^{2}}\right]^{\frac{1}{3-k}}, \quad \frac{R_{\mathrm{dec}}(\theta)}{R_{\mathrm{dec}, \mathrm{c}}}=\Theta^{\frac{2 b-a}{3-k}}$.

A slightly more complex expression than equation (2), that is valid also for $\Gamma \gtrsim 1$ and self-consistently accounts for energy conservation is given by (Panaitescu \& Kumar 2000; Gill \& Granot 2018)

$$
\begin{aligned}
\Gamma(\theta, R)= & \frac{\Gamma_{0}(\theta)+1}{2} \zeta^{k-3} \\
& \times\left[\sqrt{1+\frac{4 \Gamma_{0}(\theta)}{\Gamma_{0}(\theta)+1} \zeta^{3-k}+\left(\frac{2 \zeta^{3-k}}{\Gamma_{0}(\theta)+1}\right)^{2}}-1\right]
\end{aligned}
$$

Both equations (2) and (4) hold as long as the dynamics are completely radial, i.e. in the limit of no lateral expansion, since for simplicity we assume that $\epsilon(\theta)$ does not evolve with time and at each $\theta$ the flow behaves as if it were part of a spherical flow with the local value of $\epsilon(\theta)$. This approximation is expected to hold so long as ${ }^{1} \Gamma_{\mathrm{c}}>\theta_{\mathrm{c}}^{-1}$, as before that point the core of the jet is causally disconnected from the wings. Beyond that radius the jet can begin to expand sideways, causing the radial velocity to decrease and the energy structure to be modified.

The degree to which lateral spreading occurs is still a topic of debate, and different formulations have been proposed in the literature (e.g. Panaitescu \& Mészáros 1999; Rhoads 1999; Sari, Piran \& Halpern 1999; Granot \& Piran 2012), which apply to a tophat jet rather than a structured jet. For the sake of clarity and to avoid the uncertainties that are involved in the expectations from lateral spreading we focus here on the situation where the lateral spreading is negligible (in particular, this enables us to directly compare the results to semi-analytic models). The general expectation is that if jet's lateral spreading becomes important, it will mostly affect observers at large viewing angles, for which it would cause the main peak of the light curve to occur earlier, with a steeper rise leading to this peak and a steeper decay following it.

By integrating equation (4), we can find the (source frame) emission time $t_{\mathrm{s}}(\theta, R)=\int_{0}^{R} \mathrm{~d} R^{\prime} /\left(c \beta\left(\theta, R^{\prime}\right)\right)$, where $\beta(\theta, R) \equiv$ $\sqrt{1-\Gamma(\theta, R)^{-2}}$ is the normalized velocity corresponding to $\Gamma(\theta$, $R)$. The source frame time is related to the observer time via the light traveltime from different locations and for points in the jet that are along the line connecting the jet axis and the LOS is given by

$t=t_{\mathrm{s}}-R \cos \left(\theta_{\mathrm{obs}}-\theta\right) / c$,

where here and in what follows, we omit redshift corrections for clarity (these can be trivially added retrospectively and in any case are expected to be small for off-axis events discussed here). For an observer within the beaming cone of the material from each angle $\theta\left(\left|\theta_{\text {obs }}-\theta\right| \lesssim 1 / \Gamma(\theta, t)\right), t(\theta, R)=t_{\mathrm{s}}(\theta, R) / 2 \Gamma(\theta, R)^{2}$. We define the apparent deceleration times for such observers

$t_{\mathrm{dec}, \mathrm{c}}=\frac{R_{\mathrm{dec}, \mathrm{c}}}{2 c \Gamma_{\mathrm{c}, 0}^{2}}, \quad \frac{t_{\mathrm{dec}}(\theta)}{t_{\mathrm{dec}, \mathrm{c}}}=\Theta^{\frac{2(4-k) b-a}{3-k}}$.

\footnotetext{
${ }^{1}$ More accurately, the local condition that allows for significant lateral expansion from causality considerations is $\Gamma \theta<1$. This may occur in some parts of the jet, while others are still 'frozen' to their initial energy. As long as the jet is relativistic then the change in $\epsilon(\theta)$ is typically not very large and can still be neglected (Kumar \& Granot 2003).
} 
Table 1. Some useful notations considered in this paper.

\begin{tabular}{|c|c|c|}
\hline Notation & Definition & $\begin{array}{l}\text { Relevant } \\
\text { equation }\end{array}$ \\
\hline$\theta_{\mathrm{c}}$ & Half-opening angle of the jet's core & - \\
\hline$\theta_{\text {obs }}$ & Observer's viewing angle (relative to jet axis) & - \\
\hline$q$ & Normalized observer's viewing angle, $\theta_{\mathrm{obs}} / \theta_{\mathrm{c}}$ & - \\
\hline$\Theta$ & $\sqrt{1+q^{2}}$ & 1 \\
\hline$\theta_{F}(t)$ & Polar angle of matter dominating $F_{v}(t)$ & 9 \\
\hline$\theta_{\min }(t)$ & Lowest latitude within $\Gamma^{-1}\left(\theta_{\min }\right)$ from observer & 8 \\
\hline$\theta_{*}$ & Lowest latitude initially beamed to the observer & 17 \\
\hline$\theta_{\text {beam }}(t)$ & Polar angle equal to its beaming angle, $\Gamma \theta=1$ & 22 \\
\hline$\theta_{\mathrm{dec}}(t)$ & Latitude decelerating at $t$ & 20 \\
\hline$\epsilon_{\mathrm{c}}$ & Jet core's initial kinetic energy per solid angle & 1 \\
\hline$\Gamma_{\mathrm{c}, 0}$ & Jet core's initial Lorentz factor & 1 \\
\hline$\xi_{\mathrm{c}}$ & $\left(\Gamma_{\mathrm{c}, 0} \theta_{\mathrm{c}}\right)^{2}$ & - \\
\hline$\zeta$ & $R / R_{\operatorname{dec}}(\theta)$ & - \\
\hline$a$ & Jet's energy angular slope: $-\mathrm{d} \log \epsilon / \mathrm{d} \log \Theta$ & 1 \\
\hline$b$ & $\begin{array}{l}\text { Initial specific kinetic energy angular slope: } \\
\qquad-\operatorname{dlog}\left(\Gamma_{0}-1\right) / d \log \Theta\end{array}$ & 1 \\
\hline$k$ & External density PL index: $\rho=A R^{-k}$ & - \\
\hline$t_{\mathrm{dec}, \mathrm{c}}$ & Apparent deceleration time of the jet's core & 6 \\
\hline$\tilde{t}$ & Normalized (apparent) time, $t / t_{\mathrm{dec}, \mathrm{c}}$ & - \\
\hline$\tilde{t}_{*}$ & $\tilde{t}_{\mathrm{dec}}\left(\theta_{*}\right)$ & 21 \\
\hline$\tilde{t}_{\mathrm{c}}$ & $\Gamma_{\mathrm{c}}\left(\tilde{t}_{\mathrm{c}}\right)=\theta_{\mathrm{c}}^{-1}$ & - \\
\hline$\tilde{t}_{\mathrm{pk}}$ & Normalized time of main peak & 31 \\
\hline$\tilde{t}_{1 \mathrm{pk}}$ & Normalized time of early peak & 32 \\
\hline$\tilde{t}_{\text {dip }}$ & Normalized time of dip & 33 \\
\hline$F_{\mathrm{pk}}$ & Flux density of main peak & 40 \\
\hline$F_{1 \mathrm{pk}}$ & Flux density of early peak & 41 \\
\hline
\end{tabular}

After the deceleration time and while the flow is still relativistic $\left(t_{\mathrm{dec}}(\theta)<t<t_{\mathrm{NR}}(\theta)\right)$ we obtain an approximation for a PL jet,

$\Gamma(\theta, t)=\Gamma_{\mathrm{c}, 0}\left(\frac{t}{t_{\mathrm{dec}, \mathrm{c}}}\right)^{\frac{k-3}{8-2 k}}\left[1+\left(\frac{\theta}{\theta_{\mathrm{c}}}\right)^{2}\right]^{\frac{-a}{4(4-k)}}=\Gamma_{\mathrm{c}, 0} \tilde{t}^{\frac{k-3}{8-2 k}} \Theta^{\frac{-a}{8-2 k}}$,

where $\tilde{t} \equiv t / t_{\mathrm{dec}, \mathrm{c}}$.

\section{REGIONS DOMINATING THE OBSERVED EMISSION}

For energy structures that are reasonably steep $(a \gtrsim 2)$, more inner regions of the jet, that have lower $\theta$, can potentially result in larger contributions to the emission (provided that their radiation is not beamed away from the observer and that the material there has begun decelerating and therefore radiating significantly). It is therefore constructive to define two characteristic angles: (i) $\theta_{\min }\left(t, \theta_{\text {obs }}\right)$ as the minimal polar angle that becomes visible to an observer at $\theta_{\mathrm{obs}}$ (i.e. the observer enters the $\Gamma^{-1}$ beaming cone from $\theta_{\text {min }}$ ) at time $t$ (following Gill \& Granot 2018), and (ii) $\theta_{F}\left(t, \theta_{\text {obs }}\right)$ as the angle that dominates the contribution to the flux received by an observer at $\theta_{\text {obs }}$ at time $t$ (Takahashi \& Ioka 2019). A summary of all the characteristic angles and other notations in the problem is provided in Table 1.

The angle $\theta_{\min }\left(t, \theta_{\mathrm{obs}}\right)$ is given by

$\theta_{\mathrm{obs}}-\theta_{\min }=\frac{1}{\Gamma\left(\theta_{\min }, t\right)}=\left[\frac{2^{3-k} A c^{5-k} t^{3-k}}{(3-k) \epsilon_{\mathrm{c}}}\right]^{\frac{1}{8-2 k}} \Theta_{\min }^{\frac{a}{8-2 k}}$

where the second equality is valid for a PL jet and $t>t_{\mathrm{dec}}\left(\theta_{\min }\right)$.
To find $\theta_{F}\left(\theta_{\mathrm{obs}}, t\right)$ one needs to maximize the contribution to $\mathrm{d} F_{v}\left(\theta_{\text {obs }}, t\right) / \mathrm{d} \Omega$ as a function of $\theta$. Because of azimuthal symmetry, the corresponding brightest point of the jet is always along the line connecting the jet axis and the LOS, so we can use equation (5), and more generally also $\hat{n} \cdot \hat{\beta}=\hat{n} \cdot \hat{r}=\cos \tilde{\theta}=\cos \left(\theta_{\text {obs }}-\theta\right)$, where $\tilde{\theta}$ is the angle from the LOS. Recall that $\mathrm{d} F_{v} / \mathrm{d} \Omega \propto \mathcal{D}^{3} \frac{\mathrm{d} L^{\prime}}{\mathrm{d} v^{\prime}}$ where $\mathcal{D}(\theta, t)=\left[\Gamma\left(1-\beta \cos \left(\theta_{\mathrm{obs}}-\theta\right)\right)\right]^{-1}$ is the Doppler factor and $\frac{\mathrm{d} L^{\prime}}{\mathrm{d} v^{\prime}}(\theta, t)$ is the spectral luminosity in the jet's comoving frame. For a PL spectrum in the comoving frame: $\frac{\mathrm{d} L^{\prime}}{\mathrm{d} v^{\prime}} \propto\left(v^{\prime}\right)^{-\beta_{\nu}}$, one obtains $\mathrm{d} F_{v} /\left.\mathrm{d} \Omega \propto \mathcal{D}^{3+\beta_{v}} \frac{\mathrm{d} L^{\prime}}{\mathrm{d} v^{\prime}}\right|_{v}$. Assuming the spectrum to be dominated by synchrotron radiation from the forward shock, we can express $\left.\frac{\mathrm{d} L^{\prime}}{\mathrm{d} v^{\prime}}\right|_{\nu}$ in terms of $\epsilon, R$, and $\beta_{v}$ in terms of $p$ (the slope of the accelerated electrons energy PL distribution, with $\mathrm{d} N / \mathrm{d} \gamma_{\mathrm{e}} \propto \gamma_{\mathrm{e}}^{-p}$ for $\gamma_{\mathrm{e}}>\gamma_{\mathrm{m}}$ ). For example for $R>R_{\operatorname{dec}}(\theta)$ and $v_{\mathrm{c}}>v_{\mathrm{m}}$, we obtain ${ }^{2}$

$$
\frac{\mathrm{d} F_{v}(\theta, R)}{\mathrm{d} \Omega} \propto \begin{cases}\mathcal{D}^{8 / 3} R^{3-4 k / 3} & v_{\mathrm{a}}<v<v_{\mathrm{m}}, \\ \mathcal{D}^{\frac{5+p}{2}} \epsilon^{\frac{3 p-1}{4}} R^{[15-9 p-2 k(3-p)] / 4} & v_{\mathrm{m}}<v<v_{\mathrm{c}} \\ \mathcal{D}^{\frac{6+p}{2}} \epsilon^{\frac{3 p-2}{4}} R^{[14-9 p+2 k(p-2)] / 4} & v_{\mathrm{m}}, v_{\mathrm{c}}<v,\end{cases}
$$

where $v_{\mathrm{a}}$ is the synchrotron self-absorption frequency, $v_{\mathrm{m}}$ is the synchrotron emission frequency of minimal energy $\left(\gamma_{\mathrm{e}}=\gamma_{\mathrm{m}}\right)$ electrons, and $v_{\mathrm{c}}$ is that of electrons that cool on the dynamical time. We obtain an approximation for $\theta_{\mathrm{F}}\left(\theta_{\mathrm{obs}}, t\right)$ in the following way. We first find $\theta_{\mathrm{F}}(R)$ by maximizing $\mathrm{d} F_{v}(\theta, R) / \mathrm{d} \Omega$ over $\theta$. We then relate $R$ and $\theta_{\mathrm{F}}(R)$ to the observer frame using the relation for the equal arrival time given in equation (5), $t=t_{\mathrm{s}}\left(\theta_{\mathrm{F}}(R), R\right)-R \cos \left(\theta_{\mathrm{obs}}\right.$ $\left.-\theta_{\mathrm{F}}(R)\right) / c$. This is an approximation, as we are maximizing for a constant $R$ rather than a constant $t$ or a full integration over the equal arrival time surface. This procedure can be easily and rapidly evaluated numerically. The goodness of this approximation can be evaluated by comparing to the numerical model presented in Gill \& Granot (2018, hereafter GG18). The latter involves a full integration of the flux over the entire jet surface at all emission times and frequencies.

A comparison of our approximations for $\theta_{\mathrm{F}}(t), \theta_{\min }(t)$ to the values extracted from the full integration from the calculation of GG18 is shown in Fig. 1. We also present the angular maps showing the strength of $\mathrm{d} F_{\nu} / \mathrm{d} \tilde{\Omega}$, where $\mathrm{d} \tilde{\Omega}$ is the unit solid angle measured around the LOS, in Fig. 2. This figure demonstrates that at early and/or late times the image of the source is roughly spherical around $\theta_{\mathrm{F}}(t)$, while at intermediate times, the flux contours tend to deviate from the spherical assumption that we make later. We return to address the importance of this fact in Section 4.

Both $\theta_{\mathrm{F}}(t), \theta_{\min }(t)$ are initially roughly constant, with $\theta_{\min , 0}<$ $\theta_{\mathrm{F}, 0}<\theta_{\mathrm{obs}}$, where $\theta_{\min }(t=0) \equiv \theta_{\min , 0}, \theta_{\mathrm{F}}(t=0) \equiv \theta_{\mathrm{F}, 0}$.

One may be obtain $\theta_{\min , 0}$ by replacing $\Gamma$ with $\Gamma_{0}$ in equation (8) which for $^{3} \Gamma_{0}\left(\theta_{\min , 0}\right) \gg 1$ yields

$\xi_{\mathrm{c}}(q-x)^{2}=\left(1+x^{2}\right)^{b}, \quad x \equiv \theta_{\min , 0} / \theta_{\mathrm{c}}$.

For $b=1,2$, this has the relatively simple analytic solutions,

${ }^{2}$ This is expected to be the case starting from relatively early times. Similar expressions can be found for the three other cases, following the expressions for $\left.\frac{\mathrm{d} L^{\prime}}{\mathrm{d} v^{\prime}}\right|_{\nu}$ as a function of $\zeta$ as detailed in Granot (2005) and Table 2.

${ }^{3}$ This condition is required in order for relativistic beaming to be important early on, and for the approximation of the beaming-cone half-opening angle as $1 / \Gamma$ to hold. Note that depending on the jet structure, the limit $\Gamma_{0}\left(\theta_{\min , 0}\right)$ $\gg 1$ may not hold for large viewing angles. 


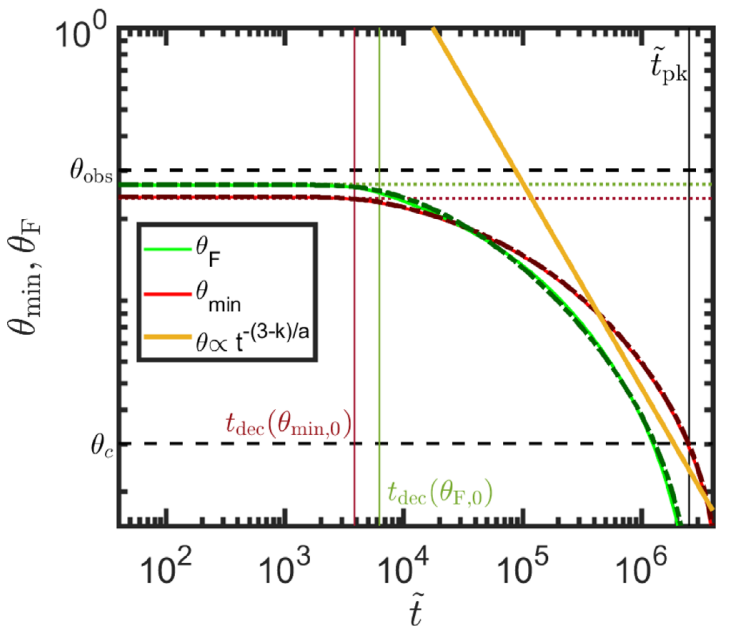

Figure 1. Temporal evolution of the angle from which the observed flux is dominated $\left(\theta_{\mathrm{F}}(t)\right.$; green $)$ and the minimal angle at the edge of the beaming cone from the observer $\left(\theta_{\min }(t)\right.$; red) as a function of $\tilde{t} \equiv t / t_{\mathrm{dec}, \mathrm{c}}$. Dotteddashed lines depict the numerical approximations based on the formulation presented in Section 3, while solid lines depict the results extracted from the numerical model of GG18. Horizontal dashed lines depict the estimates to $\theta_{\mathrm{F}, 0}, \theta_{\mathrm{min}, 0}$ given by equations (13) and (11), respectively. A solid yellow line depicts the approximate PL evolution expected at late times (equation 16). All cases are plotted for $\Gamma_{\mathrm{c}}=1000, \theta_{\mathrm{c}}=0.03, a=4, b=2$, $\theta_{\text {obs }}=0.3, k=0, p=2.2$, and assuming power-law segment (PLS) G (see Granot \& Sari 2002) for the synchrotron emission.

$$
\begin{aligned}
& \frac{\theta_{\min , 0}}{\theta_{\mathrm{c}}}=\frac{\xi_{\mathrm{c}} q-\sqrt{\xi_{\mathrm{c}} q^{2}+\xi_{\mathrm{c}}-1}}{\xi_{\mathrm{c}}-1} \text { for } b=1 \\
& \frac{\theta_{\min , 0}}{\theta_{\mathrm{c}}}=\frac{1}{2}\left(\sqrt{4 \sqrt{\xi_{\mathrm{c}}} q+\xi_{\mathrm{c}}-4}-\sqrt{\xi_{\mathrm{c}}}\right) \text { for } b=2
\end{aligned}
$$

For $\theta_{\mathrm{F}, 0}$, the equation in the ultrarelativistic limit is given by

$$
(2 b-\Lambda)(q-y)^{2}+2 \frac{1+y^{2}}{y}(q-y)=\frac{\Lambda}{\xi_{\mathrm{c}}}\left(1+y^{2}\right)^{b}, \quad y \equiv \frac{\theta_{\mathrm{F}, 0}}{\theta_{\mathrm{c}}}
$$

where

$\Lambda=b+a \frac{\lambda_{\epsilon}}{\lambda_{\mathcal{D}}}$

and $\lambda_{\epsilon}, \lambda_{\mathcal{D}}$ are the PL exponents of $\epsilon, \mathcal{D}$ respectively in equation (9). For example, for $\zeta>1, v_{\mathrm{m}}<v<v_{\mathrm{c}}$ (PLS G of Granot \& Sari 2002), we get $\Lambda=b+\frac{a(3 p-1)}{2(5+p)}$. A list of values relevant for other regimes is given in Table 2. Approximate solutions to equation (13) can be given in two limiting cases, depending on the value of $\theta_{\text {obs }}$ relative to the critical angle $\theta_{*}=\theta_{\mathrm{c}} \xi_{\mathrm{c}}^{\frac{1}{2(b-1)}}$ (see equation 17 and Section 4) for a physical interpretation of $\theta_{*}$ ). The result is $\theta_{\mathrm{F}, 0} \approx \theta_{\mathrm{obs}}$ for $\theta_{\mathrm{obs}} \ll \theta_{*}$ and $\theta_{\mathrm{F}, 0} \approx \theta_{*}\left(\theta_{\mathrm{obs}} / \theta_{*}\right)^{1 / b}$ for $\theta_{\mathrm{obs}} \gg \theta_{*}$ (these two limits can be understood intuitively, see Section 4.1). One can combine these limits into an approximation that can be used without prior knowledge of $\theta_{\mathrm{F}, 0}$ :

$\theta_{\mathrm{F}, 0}=\left[\theta_{\mathrm{obs}}^{-s}+\left(\theta_{*}\left(\frac{\theta_{\mathrm{obs}}}{\theta_{*}}\right)^{\frac{1}{5}}\right)^{-s}\right]^{-1 / s}$

where $s>0$ is a smoothing parameter that ensures the transition between the appropriate approximations at $\theta_{\mathrm{obs}} \approx \theta_{*}$. A choice that matches well the exact solution is $s=1.5$.

The angles $\theta_{\min }$ and $\theta_{\mathrm{F}}$ start decreasing significantly at $t_{\mathrm{dec}}\left(\theta_{\min , 0}\right)$ and $t_{\mathrm{dec}}\left(\theta_{\mathrm{F}, 0}\right)$, respectively. In the limit $\theta_{\mathrm{obs}} \gg \theta_{\mathrm{F}}, \theta_{\min } \gg \theta_{\mathrm{c}}$ and as long as the flow is still relativistic, both angles decrease as a PL with time. Using equation (8) and approximating $\theta_{\mathrm{obs}}-\theta_{\min } \approx \theta_{\mathrm{obs}}$, we
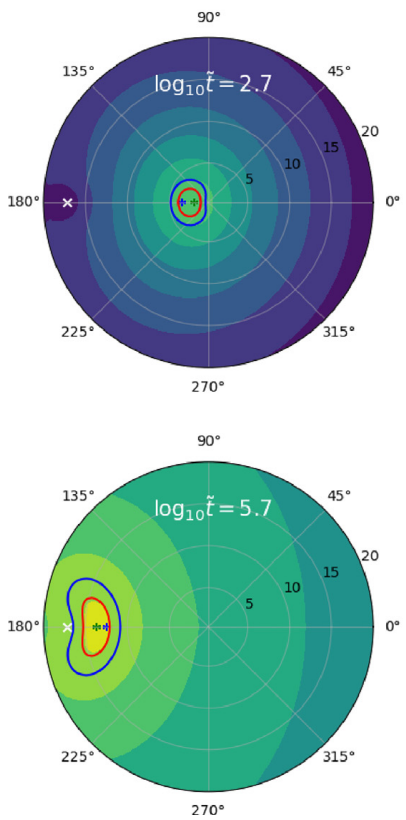
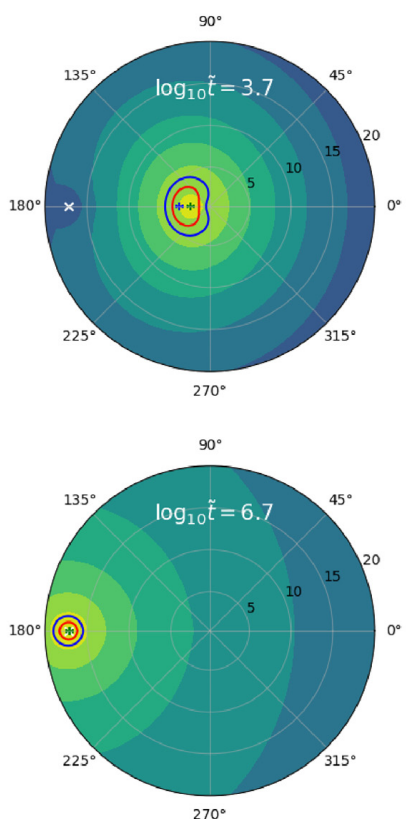
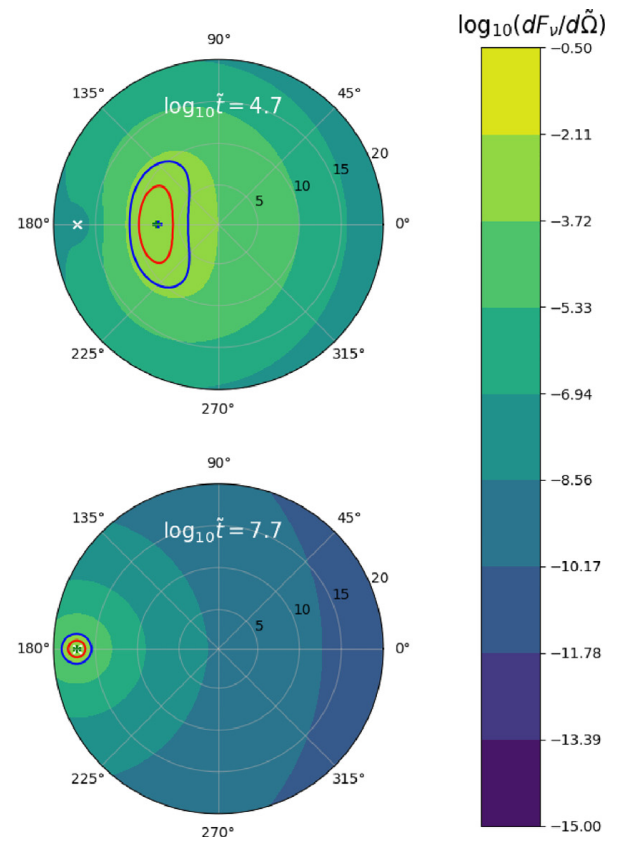

Figure 2. Angular map of $\mathrm{d} F_{\nu} / \mathrm{d} \tilde{\Omega}$ at different times, where $\mathrm{d} \tilde{\Omega}$ denotes the solid angle centred around the LOS to the observer. A white cross marks the axis of the jet. The peak of $\mathrm{d} F_{v} / \mathrm{d} \tilde{\Omega}(\tilde{t})$ is $\theta_{\mathrm{obs}}-\theta_{\mathrm{F}}(\tilde{t})$ and is marked with a green ' + ' sign. Red and blue contours contain angular regions contributing 50 per cent and 80 per cent of the total flux, respectively. For comparison, we show with a blue ' + ' sign the value of $\theta_{\text {obs }}-\theta_{\min }(\tilde{t})$. Results are plotted using the method outlined in GG18 for $\Gamma_{\mathrm{c}}=1000, \theta_{\mathrm{c}}=0.03, a=4, b=2, \theta_{\mathrm{obs}}=0.3, k=0, p=2.2$, and for a frequency such that the emission is dominated by PLS G of the synchrotron emission. 
Table 2. Value of $\Lambda$ as defined by equation (14) for different ranges of $\zeta=R / R_{\operatorname{dec}}(\theta)$ and observed synchrotron PLSs, using the notations introduced by Granot \& Sari (2002) in brackets. We also quote the spectral index in each PLS $\left(\beta_{v}\right)$ and the temporal index $\alpha_{i}$ for a spherical outflow $\left(\alpha_{\mathrm{r}}\right.$ for $\zeta<1$ or $\alpha_{\mathrm{d}}$ for $\left.\zeta>1\right)$.

\begin{tabular}{|c|c|c|c|c|c|c|}
\hline$\Lambda$ & $\zeta$ & PLS & $\beta_{v}$ & $\alpha_{i}$ & $\lambda_{\mathcal{D}}$ & $\lambda_{\epsilon}$ \\
\hline$b$ & $\zeta>1$ & $v_{\mathrm{a}}<v<v_{\mathrm{m}}<v_{\mathrm{c}}(\mathrm{D})$ & $-1 / 3$ & $\frac{2-k}{4-k}$ & $8 / 3$ & 0 \\
\hline$b+\frac{a}{4}$ & $\zeta>1$ & $v_{\mathrm{a}}<v<v_{\mathrm{c}}<v_{\mathrm{m}}(\mathrm{E})$ & $-1 / 3$ & $\frac{2-3 k}{3(4-k)}$ & $8 / 3$ & $2 / 3$ \\
\hline$b+\frac{a}{14}$ & $\zeta>1$ & $v_{\mathrm{c}}<v<v_{\mathrm{m}}(\mathrm{F})$ & $1 / 2$ & $-1 / 4$ & $7 / 2$ & $1 / 4$ \\
\hline$b+\frac{a(3 p-1)}{2(5+p)}$ & $\zeta>1$ & $v_{\mathrm{m}}<v<v_{\mathrm{c}}(\mathrm{G})$ & $(p-1) / 2$ & $\frac{k(3 p-5)-12(p-1)}{4(4-k)}$ & $\frac{p+5}{2}$ & $\frac{3 p-1}{4}$ \\
\hline$b+\frac{a(3 p-2)}{2(6+p)}$ & $\zeta>1$ & $v>\max \left(v_{\mathrm{m}}, v_{\mathrm{c}}\right)(\mathrm{H})$ & $p / 2$ & $(2-3 p) / 4$ & $\frac{p+6}{2}$ & $\frac{3 p-2}{4}$ \\
\hline$b$ & $\zeta<1$ & $v_{\mathrm{a}}<v<v_{\mathrm{m}}<v_{\mathrm{c}}(\mathrm{D})$ & $-1 / 3$ & $3-k / 2$ & $8 / 3$ & 0 \\
\hline$b$ & $\zeta<1$ & $v_{\mathrm{a}}<v<v_{\mathrm{c}}<v_{\mathrm{m}}(\mathrm{E})$ & $-1 / 3$ & $11 / 3-2 k$ & $8 / 3$ & 0 \\
\hline$b$ & $\zeta<1$ & $v_{\mathrm{c}}<v<v_{\mathrm{m}}(\mathrm{F})$ & $1 / 2$ & $2-3 k / 4$ & $7 / 2$ & 0 \\
\hline$b$ & $\zeta<1$ & $v_{\mathrm{m}}<v<v_{\mathrm{c}}(\mathrm{G})$ & $(p-1) / 2$ & $3-k(p+5) / 4$ & $\frac{p+5}{2}$ & 0 \\
\hline$b$ & $\zeta<1$ & $v>\max \left(v_{\mathrm{m}}, v_{\mathrm{c}}\right)(\mathrm{H})$ & $p / 2$ & $2-k(p+2) / 4$ & $\frac{p+6}{2}$ & 0 \\
\hline
\end{tabular}

Table 3. Summary of light-curve types in the different cases discussed in this paper.

\begin{tabular}{lccccc}
\hline Case & Condition & Number of peaks & Time-scales & $\theta_{\text {dec }}(t)$ & Approx. $\theta_{\text {min, } 0}, \theta_{\mathrm{F}, 0}$ \\
\hline $1 \mathrm{~A}$ & $\xi_{\mathrm{c}}>1, b>b_{\mathrm{c}}>b_{\mathrm{a}}, \theta_{\mathrm{obs}}<\theta_{*}$ & 2 & $t_{\mathrm{dec}}\left(\theta_{\mathrm{obs}}\right)<t_{\text {beam }}\left(\theta_{\mathrm{obs}}\right)<t_{*}$ & Increasing & $\theta_{\min , 0} \approx \theta_{\mathrm{F}, 0} \approx \theta_{\mathrm{obs}}$ \\
$1 \mathrm{~B}$ & $\xi_{\mathrm{c}}>1, b>b_{\mathrm{c}}>b_{\mathrm{a}}, \theta_{\mathrm{obs}}>\theta_{*}$ & 1 & $t_{\mathrm{dec}}\left(\theta_{\mathrm{obs}}\right)>t_{*}$ & Increasing & $\theta_{\min , 0} \approx \theta_{\mathrm{F}, 0} \ll \theta_{\mathrm{obs}}$ \\
2 & $\xi_{\mathrm{c}}>1, b_{\mathrm{c}}>b>b_{\mathrm{a}}$ & 2 & $t_{\mathrm{dec}}\left(\theta_{\mathrm{obs}}\right)<t_{\text {beam }}\left(\theta_{\mathrm{obs}}\right)$ & Increasing & $\theta_{\min , 0} \approx \theta_{\mathrm{F}, 0} \approx \theta_{\mathrm{obs}}$ \\
3 & $\xi_{\mathrm{c}}>1, b_{\mathrm{c}}>b_{\mathrm{a}}>b$ & 2 & $t_{\mathrm{dec}}\left(\theta_{\mathrm{obs}}\right)<t_{\mathrm{beam}}\left(\theta_{\mathrm{obs}}\right)$ & Declining & $\theta_{\min , 0} \approx \theta_{\mathrm{F}, 0} \approx \theta_{\mathrm{obs}}$ \\
\hline
\end{tabular}

find an asymptotic behaviour

$\theta_{\min } \approx \theta_{\mathrm{c}}\left(\frac{\tilde{t}}{\tilde{t}_{\mathrm{pk}}}\right)^{\frac{k-3}{a}} \approx \theta_{\mathrm{obs}}\left(\frac{\tilde{t}}{\tilde{t}_{\mathrm{dip}}}\right)^{\frac{k-3}{a}} \approx \theta_{*}\left(\frac{\tilde{t}}{\tilde{t}_{*}}\right)^{\frac{k-3}{a}} \propto \tilde{t}^{\frac{-(3-k)}{a}}$,

where $\tilde{t}_{\mathrm{pk}} \approx\left(\xi_{\mathrm{c}} q^{2}\right)^{(4-k) /(3-k)}$ is the time of the main peak of the light curve (i.e. the latter one, if there are two peaks), $\tilde{t}_{\text {dip }} \approx \xi_{\mathrm{c}}^{(4-k) /(3-k)} q^{[2(4-k)-a] /(3-k)}$ is the time of the dip in the light curve (in case it is double-peaked, see Section 4 ), and $\tilde{t}_{*} \approx$ $\xi_{\mathrm{c}}^{[2(4-k) b-a] /[2(b-1)(3-k)]}$ is the deceleration time of the lowest latitude initially beamed at the observer (see Section 4 for more details). As shown in Figs 1 and 2, $\theta_{\mathrm{F}}(\tilde{t})$ follows a similar asymptotic trend to $\theta_{\min }(\tilde{t})$.

\section{LIGHT CURVES FROM ANGULAR STRUCTURE}

The angles $\theta_{\mathrm{F}}(t), \theta_{\min }(t)$ are useful when analysing the light curve of a given burst from a fixed $\theta_{\text {obs }}$. In order to qualitatively distinguish between possible light curves seen from the same structure, but different observation angles we introduce a beaming angle and time through the relation $\Gamma \theta=1$, i.e. $t_{\text {beam }}(\theta)$ is defined through $\theta \Gamma\left[\theta, t_{\text {beam }}(\theta)\right] \equiv 1$, and the corresponding $\theta_{\text {beam }}(t)$ is defined by $\theta_{\text {beam }}(t) \Gamma\left[\theta_{\text {beam }}(t), t\right] \equiv 1$. Since there could be more than one angle that satisfies this relation, the physically relevant value of $\theta_{\text {beam }}(t)$ generally depends on the observation angle. We return to discuss the different regimes in more detail in Section 4.1.

Another critical angle is $\theta_{*}$, which is the value of $\theta$ for which the initial Lorentz factor satisfies $\theta_{*} \Gamma_{0}\left(\theta_{*}\right)=1$. It is also approximately the angle for which $\theta_{\text {beam }}(t)=\theta_{\operatorname{dec}}(t)$, which occurs at the corresponding time $t_{*} \equiv t_{\text {beam }}\left(\theta_{*}\right) \equiv t_{\text {dec }}\left(\theta_{*}\right)$ such that $\theta_{*}=\theta_{\text {beam }}\left(t_{*}\right)=$ $\theta_{\mathrm{dec}}\left(t_{*}\right)$. This is a critical value, since for $\theta \Gamma_{0}(\theta)>1$ relativistic beaming from $\theta$ is important from early on and vice versa. For $\theta_{*} \gg \theta_{\mathrm{c}}$, one can approximately write

$\theta_{*} \approx \theta_{\mathrm{c}} \xi_{\mathrm{c}}^{\frac{1}{2(b-1)}} \Longleftrightarrow q_{*} \equiv \frac{\theta_{*}}{\theta_{\mathrm{c}}} \approx \xi_{\mathrm{c}}^{\frac{1}{2(b-1)}}$

Clearly $b>1(b<1)$ is required for a declining (inclining) slope of $\Gamma_{0} \theta$ as a function of $\theta$. Furthermore, assuming that ${ }^{4} \xi_{\mathrm{c}}>1$, the existence of $\theta_{\mathrm{c}}<\theta_{*}<1$ requires $b>b_{\mathrm{c}}>1$ where

$b_{\mathrm{c}}=-\frac{\log \left(\Gamma_{\mathrm{c}, 0}\right)}{\log \left(\theta_{\mathrm{c}}\right)}=1-\frac{\log \left(\xi_{\mathrm{c}}\right)}{2 \log \left(\theta_{\mathrm{c}}\right)}$.

Another critical value of $b$ is

$b_{\mathrm{a}} \equiv \frac{a}{2(4-k)}$

(separating between jets that decelerate from the core outwards to vice versa). Different physical regimes can arise due to the different possible orderings of $b_{\mathrm{a}}, b, b_{\mathrm{c}}$. For the purposes of clarity, we assume in what follows that $b_{\mathrm{a}}<b_{\mathrm{c}}$ and explore different values of $b$. This ordering is natural, since for $\Gamma_{\mathrm{c}, 0}=200, \theta_{\mathrm{c}}=0.03$, one finds $b_{\mathrm{c}} \approx 1.5$ (and the value becomes even larger for larger values of either $\Gamma_{\mathrm{c}, 0}$ or $\left.\theta_{\mathrm{c}}\right)$. Therefore, for $k=0(k=2), a \gtrsim 12(a \gtrsim 6)$ is needed to reverse the condition assumed above. Our division to regimes is thus as follows:

(1) $b_{\mathrm{a}}<b_{\mathrm{c}}<b$ with sub-cases: $\theta_{\mathrm{obs}}<\theta_{*}(1 \mathrm{~A}) \& \theta_{\text {obs }}>\theta_{*}(1 \mathrm{~B})$,

(2) $b_{\mathrm{a}}<b<b_{\mathrm{c}}$,

(3) $b<b_{\mathrm{a}}<b_{\mathrm{c}}$.

The division to the three regimes can be related to the general behaviour of $\Gamma_{0} \theta$ as a function of $\theta$ as shown in Fig. 3. We explore

${ }^{4} \xi_{\mathrm{c}}=\left(\Gamma_{\mathrm{c}, 0} \theta_{\mathrm{c}}\right)^{2}<1$ is difficult to achieve because of the implied strong lateral causal contact during the acceleration phase, which tends to result in $\Gamma_{\mathrm{c}, 0} \theta_{\mathrm{c}} \gtrsim 1$. Moreover, interpreting bright GRBs with a jet break in the afterglow light curve as corresponding to $q=\theta_{\text {obs }} / \theta_{\mathrm{c}} \lesssim 1$, afterglow observations suggest $\Gamma_{\mathrm{c}, 0} \theta_{\mathrm{c}}$ of several to a few tens, or $\xi_{\mathrm{c}} \sim 10^{2}$. 


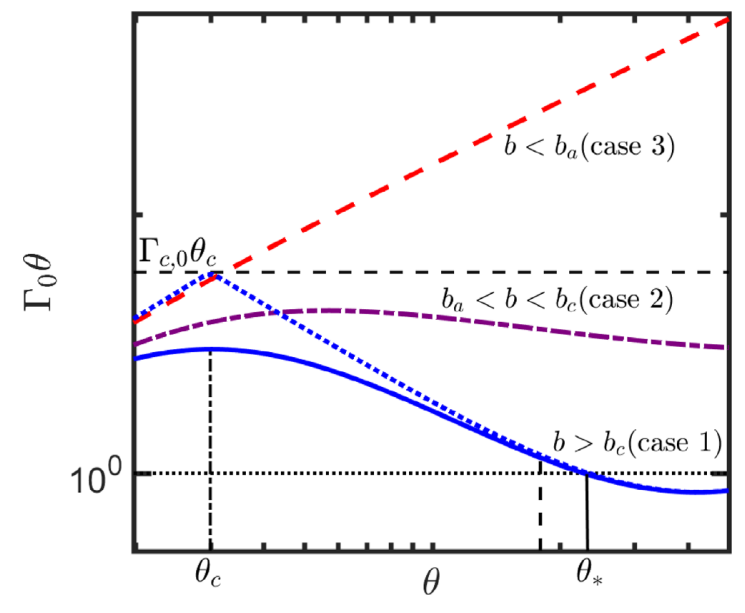

Figure 3. The profile of $\Gamma_{0} \theta$ for three different values of $b$. We have taken here: $\Gamma_{\mathrm{c}}=200, \theta_{\mathrm{c}}=0.03$ and $b=2,1.3$, and 0.2 for cases 1,2 , and 3, respectively. We also consider the case for which $b_{\mathrm{c}}>b_{\mathrm{a}}$ (see Section 4). A dashed vertical line denotes the approximate solution for $\theta_{*}$ given by equation (17) and a solid line depicts the exact value defined by $\Gamma_{0}\left(\theta_{*}\right) \theta_{*}=$ 1 . For case 1 , we also denote with a dotted blue line, the curve corresponding to the approximation $\Theta \rightarrow \max \left[1,\left(\theta / \theta_{\mathrm{c}}\right)\right]$. Additional lines depict $\Gamma_{\mathrm{c}, 0} \theta_{\mathrm{c}}$ (dashed horizontal), $\Gamma \theta=1$ (dotted horizontal), and the location of $\theta_{\mathrm{c}}$ (dotted-dashed vertical).

below the resulting light curves in each of those regimes. We focus on the case $\Theta \rightarrow \max \left[1,\left(\theta / \theta_{\mathrm{c}}\right)\right]$, in which the different PL segments can be clearly seen from the figures. We also summarize some of the important distinctions between the different cases in Table 3.

\subsection{Case 1: $\xi_{\mathrm{c}}>1, b>b_{\mathrm{c}}>b_{\mathrm{a}}$}

To understand the expected light-curve shape, it is useful to consider the orderings of the different time-scales in this regime; $\theta_{\mathrm{dec}}$ can be expressed as a simple PL with time, by inverting equation (6),

$\theta_{\mathrm{dec}}(\tilde{t})=\theta_{\mathrm{c}} \tilde{t}^{\frac{3-k}{2(4-k) b-a}}$

where $\tilde{t} \equiv t / t_{\mathrm{dec}, \mathrm{c}}$. For $\theta_{\text {beam }}$, the situation is more subtle. By definition of $\theta_{*}$, at early times, $\theta_{\text {beam }}=\theta_{*}$. This situation holds until $\tilde{t}=\tilde{t}_{\mathrm{c}}=\xi_{\mathrm{c}}^{(4-k) /(3-k)}$, which is when the core of the jet has decelerated enough that $\Gamma_{\mathrm{c}} \theta_{\mathrm{c}}=1$, i.e. $\Gamma_{\mathrm{c}}\left(\tilde{t}_{\mathrm{c}}\right)=\theta_{\mathrm{c}}^{-1}$ (note that for $b$ $>b_{\mathrm{c}}>b_{\mathrm{a}}$ deceleration occurs first at the core of the jet and only later at the wings). From this moment and until $\tilde{t}_{*}$, there are two solutions for $\theta_{\text {beam }}$, an outer solution at $\theta_{\text {out }}=\theta_{*}$ and an inner solution at $\theta_{\text {in }}=\theta_{\mathrm{c}} \xi_{\mathrm{c}}^{\frac{k-4}{2(4-k)-a}} \tilde{t}^{\frac{3-k}{2(4-k)-a}}$. Note that $\theta_{\text {in }}\left(\tilde{t}_{\mathrm{c}}\right)=\theta_{\mathrm{c}}, \theta_{\text {in }}\left(\tilde{t}_{\mathrm{dip}}\right)=\theta_{\mathrm{obs}}$ and $\theta_{\text {in }}\left(\tilde{t}_{*}\right)=\theta_{*}$ where

$\tilde{t}_{*}=\tilde{t}_{\mathrm{dec}}\left(\theta_{*}\right)=\left(\frac{\theta_{*}}{\theta_{\mathrm{c}}}\right)^{\frac{2(4-k) b-a}{3-k}}=\xi_{\mathrm{c}}^{\frac{2(4-k) b-a}{2(b-1)(3-k)}}$.

The situation changes once more at $\tilde{t}_{*}$, since beyond this time, the entire jet satisfies $\Gamma \theta<1$ and therefore $\theta_{\text {beam }}$ is no longer defined. The physically relevant $\theta_{\text {beam }}(t)$ depends on $\theta_{\text {obs }}$. For $\theta_{\mathrm{obs}}>\theta_{*}$, $\theta_{\text {in }}$ never dominates the light curve. This is because by the time material from this angle is beamed towards the observer, $\theta_{\text {beam }}$ is no longer defined (see above). Even $\theta_{\text {out }}=\theta_{*}$ is physically significant only for $\theta_{\mathrm{obs}} \approx \theta_{*}$. Alternatively, for $\theta_{\mathrm{obs}}<\theta_{*}, \theta_{\mathrm{out}}=\theta_{*}$ carries less energy than material travelling along the LOS and is never
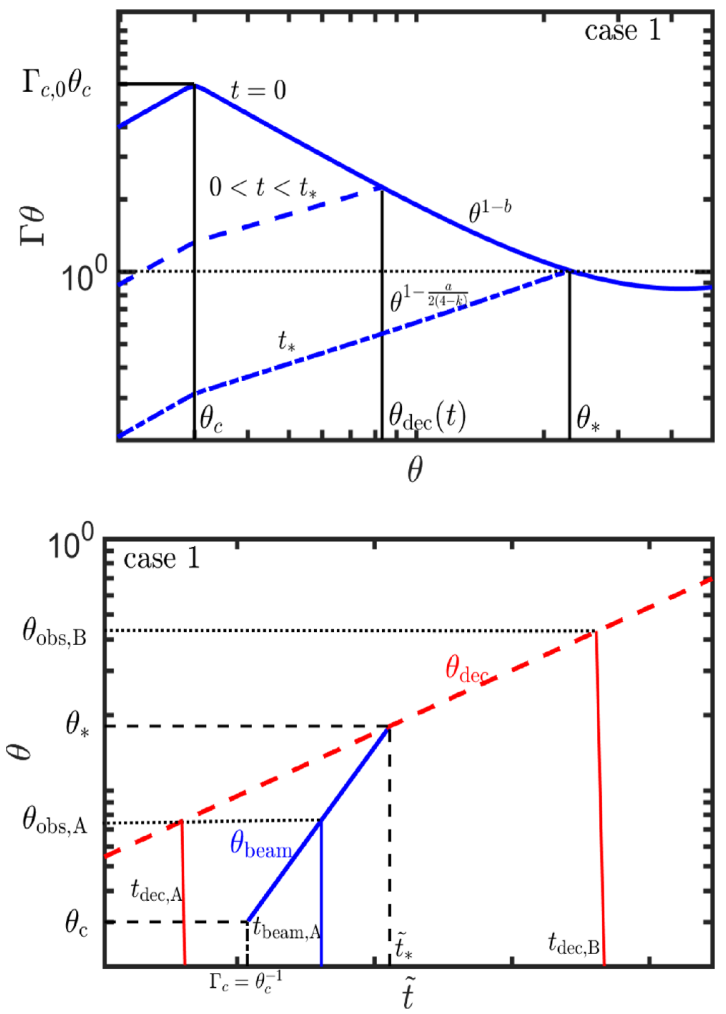

Figure 4. Top: temporal evolution of $\Gamma \theta$ in case $1\left(b>b_{\mathrm{c}}\right)$. Results are plotted for $\theta_{\mathrm{c}}=0.03, \Gamma_{\mathrm{c}}=200$ as well as $k=0, a=4$, and $b=2$. Bottom: corresponding evolution of the characteristic angles with time. For $\theta_{\mathrm{obs}}=$ $\theta_{\mathrm{obs}, \mathrm{A}}<\theta_{*}$, one gets $t_{\mathrm{dec}, \mathrm{A}}<t_{\text {beam, } \mathrm{A}}<t_{*}$ while for $\theta_{\mathrm{obs}}=\theta_{\mathrm{obs}, \mathrm{B}}>\theta_{*}$, one gets $t_{\mathrm{dec}, \mathrm{B}}>t_{*}$ (and $t_{\mathrm{beam}}$ becomes non-defined in this case).

physically important. We conclude that

$\theta_{\text {beam }}(\tilde{t})= \begin{cases}\theta_{\text {out }}=\theta_{*} & \theta_{\text {obs }}>\theta_{*}, \quad \tilde{t}<\tilde{t}_{*}, \\ \theta_{\text {in }}=\theta_{\mathrm{c}}\left(\frac{\tilde{t}^{3-k}}{\xi_{\mathrm{c}}^{4-k}}\right)^{\frac{1}{2(4-k)-a}} \theta_{\text {obs }}<\theta_{*}, \quad \tilde{t}_{\mathrm{c}}<\tilde{t}<\tilde{t}_{*},\end{cases}$

In particular, for $\tilde{t}_{\mathrm{c}}<\tilde{t}<\tilde{t}_{*}$ there are three regions in terms of the relationship between $\theta_{\text {obs }}, \theta_{\text {in }}, \theta_{\text {out }}$ which are divided as follows:

(i) $\theta_{\text {obs }}<\theta_{\text {in }}(t)-$ Here $t>t_{\text {beam }}\left(\theta_{\text {obs }}\right)$ which leads to $\theta_{\mathrm{F}} \approx \theta_{\text {min }} \ll$ $\theta_{\text {obs }}$. As we will show below this corresponds to the shallow rising phase of the light curve, $F_{v} \propto t^{\alpha}$.

(ii) $\theta_{\text {in }}(t)<\theta_{\text {obs }}<\theta_{\text {out }}=\theta_{*}-$ Here $\theta_{\mathrm{F}} \approx \theta_{\text {min }} \approx \theta_{\text {obs }}$. This represents the early (first inclining and then declining) part of the light curve, as will be detailed below.

(iii) $\theta_{\text {obs }}>\theta_{\text {out }}=\theta_{*}-$ Here $\theta_{\mathrm{F}, 0} \approx \theta_{\text {min }, 0} \approx \theta_{*}\left(\theta_{\text {obs }} / \theta_{*}\right)^{1 / b} \ll$ $\theta_{\text {obs. }}$. In this case, the behaviour changes after $t_{\mathrm{dec}}\left(\theta_{\mathrm{F}, 0}\right) \approx$ $t_{*}\left(\theta_{\mathrm{obs}} / \theta_{*}\right)^{[2(4-k) b-a] /(3-k) b}>t_{*}$, since this is when $\theta_{\mathrm{F}}, \theta_{\min }$ start to decrease significantly and the shallow rising part of the light curve, $F_{v} \propto t^{\alpha}$, emerges.

The situation is demonstrated by observing the temporal evolution of $\Gamma \theta$ and the direct evolution of $\theta_{\text {beam }}, \theta_{\mathrm{dec}}$ in Fig. 4. Evidently, two subcases exist here depending on $\theta_{\mathrm{obs}} / \theta_{*}$. We explore those subcases below. We also present the evolution of $\theta_{\mathrm{F}}\left(\theta_{\mathrm{obs}}\right)$ as a function of time in Fig. 5. This figure demonstrates the validity of our approximation for $\theta_{\mathrm{F}, 0}$, given by equation (15). For $\theta_{\mathrm{obs}} \ll$ $\theta_{*}, \theta_{\mathrm{F}, 0} \rightarrow \theta_{\text {obs }}$ as expected. For $\theta_{\mathrm{obs}} \gg \theta_{*}$, we have $\theta_{\mathrm{F}, 0} \approx \theta_{\min , 0}$ $\approx \theta_{*}\left(\theta_{\mathrm{obs}} / \theta_{*}\right)^{1 / b}$. However, in practice, for finite values of $\theta_{\mathrm{obs}} / \theta_{*}$, the real value of $\theta_{\mathrm{F}, 0}$ is slightly below the above approximation. 


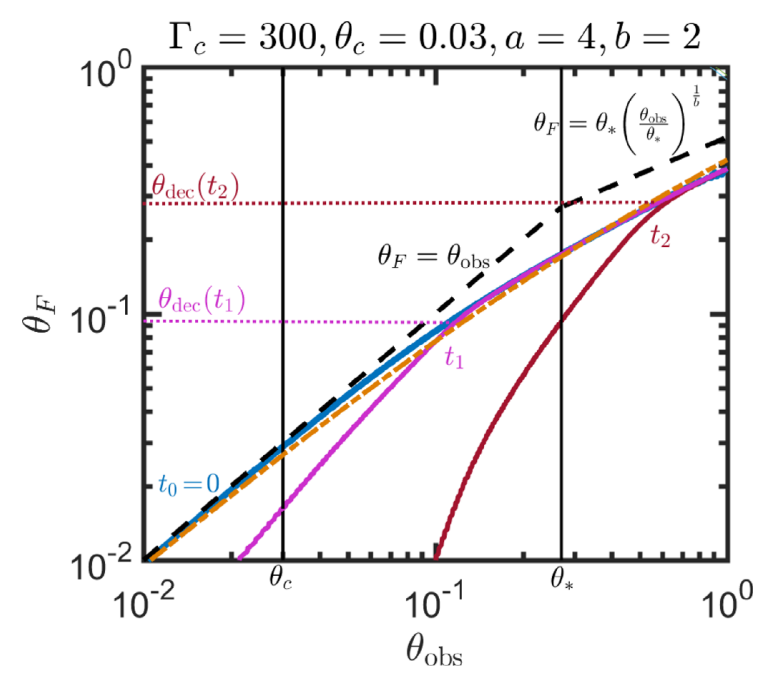

Figure 5. $\theta_{\mathrm{F}}\left(\theta_{\mathrm{obs}}\right)$ for different observation times $\left(0=t_{0}<t_{1}<t_{2}\right)$. Results are plotted for $\theta_{\mathrm{c}}=0.03, \Gamma_{\mathrm{c}}=300$ as well as $k=0, a=4$, and $b=2$. As a comparison we also plot in a dotted-dashed line the approximate value of $\theta_{\mathrm{F}, 0}$ given by equation (15), as well as the asymptotic scalings $\theta_{\mathrm{F}, 0} \approx \theta_{\mathrm{obs}}$ and $\theta_{\mathrm{F}, 0} \approx \theta_{*}\left(\theta_{\mathrm{obs}} / \theta_{*}\right)^{1 / b}$ expected to hold for $\theta_{\mathrm{obs}} \ll \theta_{*}$ and $\theta_{\mathrm{obs}} \gg \theta_{*}$, respectively, in dashed lines. Horizontal dotted lines depict $\theta_{\operatorname{dec}}\left(t_{i}\right)$ for each case as given by equation (20).

Furthermore, since the core decelerates faster than the wings, the further $\theta_{\mathrm{obs}}$ is from $\theta_{\mathrm{c}}$, the longer it takes for $\theta_{\mathrm{F}}$ to start diminishing significantly.

(i) Case 1A, $\theta_{\text {obs }}<\theta_{*}$ : here the ordering of the time-scales is $t_{\text {dec }}\left(\theta_{\text {obs }}\right)<t_{\text {beam }}\left(\theta_{\text {obs }}\right)<t_{*}$. In this case, the emission from $\theta<$ $\theta_{\text {obs }}$ is initially strongly beamed away from the observer, while the material along the LOS whose emission is beamed towards the observer lies within $\left|\theta-\theta_{\text {obs }}\right| \lesssim 1 / \Gamma\left(\theta_{\text {obs }}\right) \ll \theta_{\text {obs }}$, so the early emission is dominated by material near $\theta_{\mathrm{F}, 0} \approx \theta_{\min , 0} \approx \theta_{\text {obs. }}$. For $t<t_{\mathrm{dec}}\left(\theta_{\mathrm{F}, 0}\right)$ the flux therefore rises as $F_{v} \propto t^{\alpha_{r}}$, while the LOS material has not yet decelerated (where $\alpha_{r}$ depends on $k$ and on the observed PLS, see Tables 2 and 4). After a short, intermediate duration between $t_{\mathrm{dec}}\left(\theta_{\mathrm{F}, 0}\right), t_{\mathrm{dec}}\left(\theta_{\mathrm{obs}}\right)$, the local dynamics of the material along the LOS begin to follow a largely spherical selfsimilar evolution (Blandford \& McKee 1976) and the resulting flux is similar to cosmological GRBs viewed on-axis $F_{v} \propto t^{\alpha_{d}}$ (where e.g., for $k=0$ and PLS G $\alpha_{\mathrm{d}}=3(1-p) / 4$, see Tables 2 and 4). In practice the decay of the light curve is not as steep as in the spherical case, due to the fact that material from $\theta>\theta_{\text {obs }}$ decelerates after $t_{\mathrm{dec}}\left(\theta_{\mathrm{obs}}\right)$, and its contributions cannot be completely ignored (see Fig. 2). This phase lasts until $\tilde{t}_{\text {dip }} \approx \tilde{t}_{\text {beam }}\left(\theta_{\text {obs }}\right) \approx q^{\frac{2(4-k)-a}{3-k}} \xi_{\mathrm{c}}^{\frac{4-k}{3-k}}$ which is approximately the time when material internal to the LOS starts becoming visible as its beaming cone reaches the observer. At $t_{\mathrm{dip}}<t<t_{\mathrm{pk}}$, the emission becomes dominated by material at

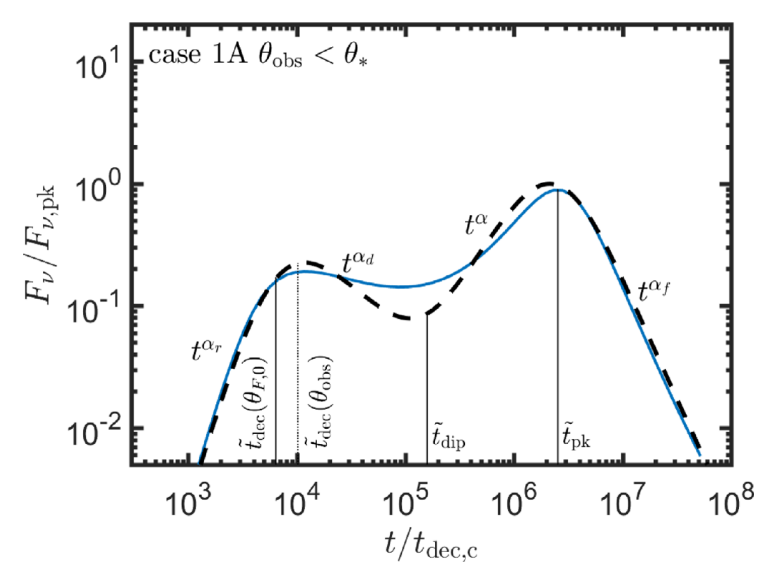

Figure 6. Analytic light curve obtained for case 1A (dashed) as compared with the numerical model of GG18 (solid). Results are shown for $k=0, p=$ 2.2, $a=4, b=2, \Gamma_{\mathrm{c}}=1000, \theta_{\mathrm{c}}=0.03, \theta_{\mathrm{obs}}=0.3$, and PLS G. $t_{\mathrm{dec}}\left(\theta_{\mathrm{F}, 0}\right)$ is calculated using equation (13).

progressively smaller $\theta \sim \theta_{\min } \sim \theta_{\mathrm{F}} \ll \theta_{\text {obs }}$ (see equation 16). This typically leads to a shallow rise in the flux, $F_{v} \propto t^{\alpha}$ (see Appendix A2 of GG18 for a derivation of the asymptotic $\alpha$ in this phase and Table 4 for the values corresponding to different PLS). The rise continues until $t_{\mathrm{pk}}$, when the jet's core becomes visible (i.e. $\theta_{\min }\left(t_{\mathrm{pk}}\right)$ $\rightarrow 0$; see Section 5). Beyond this point, the full jet becomes visible to the observer and the light curve evolves as for an on-axis GRB jet post-jet break, $F_{v} \propto t^{\alpha_{f}}$ (for a detailed discussion of this phase see e.g. Granot 2007; De Colle et al. 2012; Granot \& Piran 2012; Gill et al. 2019). To calculate the analytic light curve, the flux of each peak is calculated by

$F=\bar{F} 2^{\frac{\alpha_{1}-\alpha_{2}}{2}} \bar{t}^{\alpha_{1}}\left(1+\bar{t}^{2}\right)^{\frac{\alpha_{2}-\alpha_{1}}{2}}$

where $\bar{t}$ is the time normalized to the peak time, $\bar{F}$ is the peak flux, and $\alpha_{1}, \alpha_{2}$ are the temporal slopes before and after the peak, respectively. The overall flux is a sum of two terms of the form given by equation (23) for the two peaks, i.e.

$$
\begin{aligned}
F= & F_{1 \mathrm{pk}} 2^{\frac{\alpha_{r}-\alpha_{d}}{2}}\left(\frac{t}{t_{1 \mathrm{pk}}}\right)^{\alpha_{r}}\left[1+\left(\frac{t}{t_{1 \mathrm{pk}}}\right)^{2}\right]^{\frac{\alpha_{d}-\alpha_{r}}{2}} \\
& +F_{\mathrm{pk}} 2^{\frac{\alpha_{f}}{2}}\left(\frac{t}{t_{\mathrm{pk}}}\right)^{\alpha}\left[1+\left(\frac{t}{t_{\mathrm{pk}}}\right)^{2}\right]^{\frac{\alpha_{f}-\alpha}{2}}
\end{aligned}
$$

where $t_{1 \mathrm{pk}}, t_{\mathrm{pk}}, F_{1 \mathrm{pk}}, F_{\mathrm{pk}}$ are correspondingly the times and fluxes of the first and second fluxes. A summary of their values is given in Section 5. An illustration of the overall light curve in this case is shown in Fig. 6, side by side with the result of the numerical calculation of GG18. The analytic prescription provides a good approximation of the more complete calculation.

Table 4. Values of the temporal slopes for the different synchrotron PLS.

\begin{tabular}{lccccc}
\hline PLS & $\beta$ & $\alpha_{i}$ & $\alpha_{d}$ & $\alpha$ & $\alpha_{f}$ \\
\hline $\mathrm{D}$ & $-\frac{1}{3}$ & $3-k / 2$ & $\frac{2-k}{4-k}$ & $\frac{8(k-3)-a(16 k / 3-12)}{4 a}$ & $\frac{1}{k-4}$ \\
$\mathrm{E}$ & $-\frac{1}{3}$ & $11 / 3-2 k$ & $\frac{2-3 k}{3(4-k)}$ & $\frac{8(k-3)-a(8 k-44 / 3)}{4 a}$ & $\frac{7}{3(k-4)}$ \\
$\mathrm{F}$ & $\frac{1}{2}$ & $2-3 k / 4$ & $-1 / 4$ & $\frac{8(k-3)-a(3 k-8)}{4 a}$ & $\frac{16-5 k}{4(k-4)}$ \\
$\mathrm{G}$ & $\frac{p-1}{2}$ & $3-k(p+5) / 4$ & $\frac{k(3 p-5)-12(p-1)}{4(4-k)}$ & $\frac{8(k-3)-a((5+p) k-12)}{4 a}$ & $\frac{k+12 p-3 k p}{4(k-4)}$ \\
$\mathrm{H}$ & $\frac{p}{2}$ & $2-k(p+2) / 4$ & $(2-3 p) / 4$ & $\frac{8(k-3)-a((2+p) k-8)}{4 a}$ & $\frac{4+12 p-k(2+3 p)}{4(k-4)}$ \\
\hline
\end{tabular}




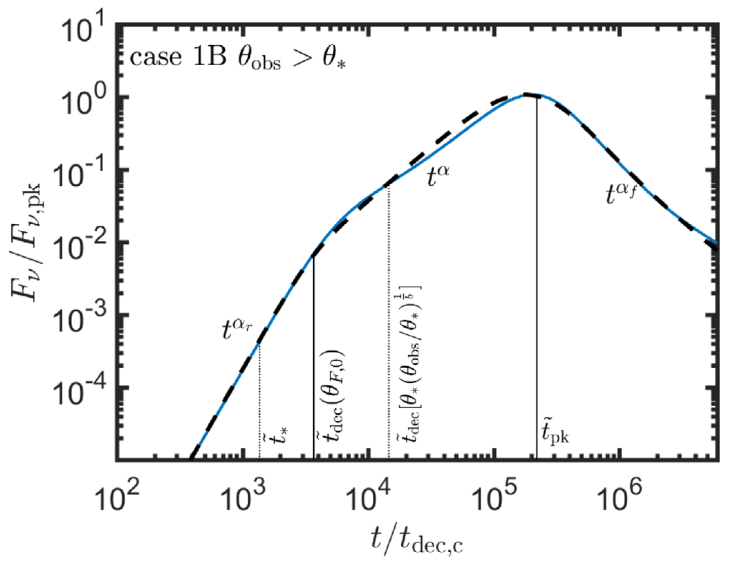

Figure 7. Analytic light curve obtained for case 1B (dashed) as compared with the numerical model of GG18 (solid). Results are shown for $k=0, p=$ $2.2, a=4, b=2, \Gamma_{\mathrm{c}}=200, \theta_{\mathrm{c}}=0.03, \theta_{\mathrm{obs}}=0.6$, and PLS G; $t_{\mathrm{dec}}\left(\theta_{\mathrm{F}, 0}\right)$ is calculated using equation (13).

(ii) Case $1 \mathrm{~B}, \theta_{\mathrm{obs}}>\theta_{*}$ : here the ordering of time-scales is $t_{*}<$ $t_{\mathrm{dec}}\left(\theta_{\mathrm{obs}}\right)$, which implies that a wide range of angles $\theta$ such that $\theta_{\mathrm{obs}}$ $\gg \theta \gtrsim \theta_{\mathrm{F}, 0}$ are visible from the very start. As a first approximation, it is constructive to consider the approximation $\Gamma_{0}^{-1}\left(\theta_{\min , 0}\right)=$ $\theta_{\text {obs }}-\theta_{\text {min }, 0} \approx \theta_{\text {obs }}$ leading to $\theta_{\mathrm{F}, 0} \approx \theta_{\text {min }, 0} \approx \theta_{\mathrm{c}}\left(\xi_{\mathrm{c}} q^{2}\right)^{1 / 2 b} \approx$ $\theta_{*}\left(\theta_{\mathrm{obs}} / \theta_{*}\right)^{1 / b} \ll \theta_{\mathrm{obs}}$, where the time of significant decrease in $\theta_{\mathrm{F}} \approx \theta_{\text {min }}$ is expected to be around $t_{\mathrm{dec}}\left(\theta_{\mathrm{F}, 0}\right) \approx t_{\mathrm{dec}}\left(\theta_{\min , 0}\right)$ $\approx\left(\xi_{\mathrm{c}} q^{2}\right)^{[2(4-k) b-a] /[2 b(3-k)]} \approx t_{*}\left(\theta_{\mathrm{obs}} / \theta_{*}\right)^{[2(4-k) b-a] /(3-k) b}>t_{*}$. However, as shown in Fig. 5, this approximation is valid only for $\theta_{\mathrm{obs}} \gg \theta_{*}$ and in practice it somewhat overestimates $\theta_{\mathrm{F}, 0}$, and correspondingly $t_{\mathrm{dec}}\left(\theta_{\mathrm{F}, 0}\right)$. For a more accurate approximation, we therefore apply equation (13). Alternatively, one can more conveniently use the analytic approximation in equation (15).

In general, the most significant contributions to the emission come from material that has both decelerated and whose emission is beamed towards the observer. At $t=t_{\mathrm{dec}}\left(\theta_{\mathrm{F}, 0}\right)$ the material from $\theta_{\mathrm{F}, 0}$ satisfies both these conditions, and indeed it dominates the observed emission at that time. At earlier times, the material at $\theta_{\mathrm{F}, 0}$, had the same $\Gamma$ as at deceleration and was therefore still beamed towards the observer, although not yet slowed down. The result is that for $t<t_{\mathrm{dec}}\left(\theta_{\mathrm{F}, 0}\right)$ the flux is still dominated by material at $\theta_{\mathrm{F}, 0}$ and rises as $F_{v} \propto t^{\alpha_{r}}$, where $\alpha_{\mathrm{r}}$ is the pre-deceleration rise of the LOS flux and depends on the spectral regime that is observed (see Table 2). At later times $t_{\mathrm{dec}}\left(\theta_{\mathrm{F}, 0}\right)<t<t_{\mathrm{pk}}$ the flux becomes dominated by $\theta \sim \theta_{\mathrm{F}}(t) \sim \theta_{\text {min }}(t)$ (see equation 16) and evolves as $F_{\nu} \propto t^{\alpha}$ as described in case $1 \mathrm{~A}$ above. The flux at $t>t_{\mathrm{pk}}$ evolves (as for 1A) according to the standard postjet break scaling. An illustration of the light curve obtained in this case is given in Fig. 7 alongside the numerical calculation of GG18. Note that emission from material along the LOS is always subdominant in this case. An expression for the flux is given by

$F=2^{\frac{\alpha-\alpha_{f}}{2}} F_{\mathrm{pk}}\left[1+\left(\frac{t}{t_{\mathrm{dec}}\left(\theta_{\mathrm{F}, 0}\right)}\right)^{-4}\right]^{\frac{\alpha-\alpha_{r}}{4}}\left(\frac{t}{t_{\mathrm{pk}}}\right)^{\alpha}\left[1+\left(\frac{t}{t_{\mathrm{pk}}}\right)^{2}\right]^{\frac{\alpha_{f}-\alpha}{2}}$

\subsection{Case 2: $\xi_{\mathrm{c}}>1, b_{\mathrm{a}}<b<b_{\mathrm{c}}$}

The expressions for $\theta_{\text {dec }}$ and $\theta_{\text {beam }}$ remain the same in this case as in case 1 above and are given by equations (20) and (22), respectively.
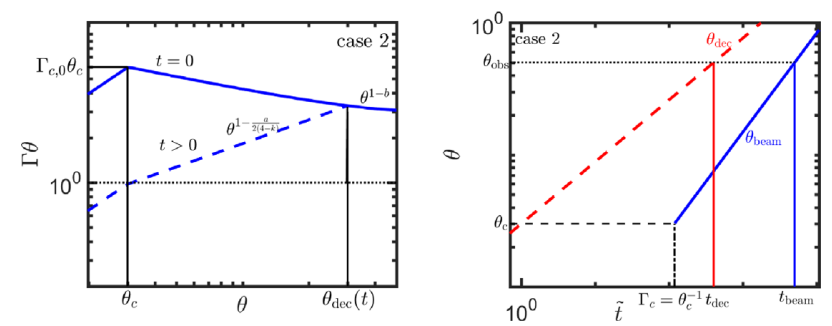

Figure 8. Left: temporal evolution of $\Gamma \theta$ in case $2\left(b_{\mathrm{a}}<b<b_{\mathrm{c}}\right)$. Results are plotted for $\theta_{\mathrm{c}}=0.03, \Gamma_{\mathrm{c}}=200$ as well as $k=0, a=4$, and $b=1.3$. Right: corresponding evolution of the characteristic angles with time. $t_{\mathrm{dec}}<$ $t_{\text {beam }}$ for any observation angle.

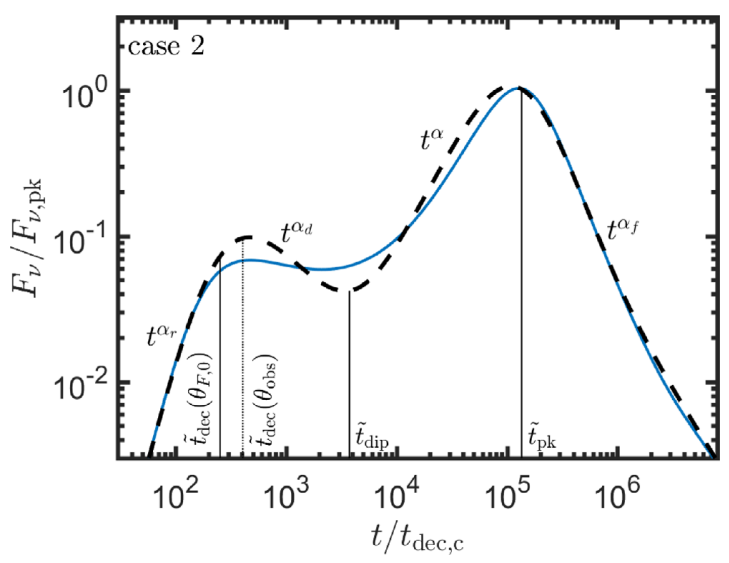

Figure 9. Analytic light curve obtained for case 2 (dashed) as compared with the numerical model of GG18 (solid). Results are shown for $k=0, p=$ 2.2, $a=4, b=1.3, \Gamma_{\mathrm{c}}=200, \theta_{\mathrm{c}}=0.03, \theta_{\mathrm{obs}}=0.5$, and PLS G. $t_{\mathrm{dec}}\left(\theta_{\mathrm{F}, 0}\right)$ is calculated using equation (13).

The difference here is that $\theta_{*}$ is no longer defined since $\theta \Gamma_{0}(\theta)$ $>1$ for all $\theta$. The resulting situation is equivalent to case $1 \mathrm{~A}$ (i.e. $b>b_{\text {c }}$ with $\left.\theta_{\text {obs }}<\theta_{*}\right)$ in which $\left.t_{\text {dec }}\left(\theta_{\text {obs }}\right)<t_{\text {beam }}\left(\theta_{\text {obs }}\right)\right)$. This is demonstrated in Fig. 8 where we plot the temporal evolution of $\Gamma \theta$ and of the critical angles. We also plot the resulting light curve in Fig. 9.

\subsection{Case 3: $\xi_{\mathrm{c}}>1, b<b_{\mathrm{a}}<b_{\mathrm{c}}$}

In this regime, the wings of the jet are sufficiently fast, that deceleration progresses from the outside in rather than vice versa as in the previous cases. As in case 2 , since $\Gamma_{0} \theta>1$ for any $\theta, \theta_{*}$ is not defined in this case. Once more, the resulting evolution is similar to cases $1 \mathrm{~A}$ and 2, i.e. $t_{\mathrm{dec}}\left(\theta_{\mathrm{obs}}\right)<t_{\text {beam }}\left(\theta_{\text {obs }}\right)$. This is shown in Fig. 10 where we plot the temporal evolution of $\Gamma \theta$ and the critical angles. We also plot the resulting light curve in Fig. 11.

\subsection{Gaussian jets}

We have focused so far on PL jets, for which closed expressions for, e.g. $\theta_{\text {beam }}(t)$ can be obtained. For completeness, we briefly discuss here the case of Gaussian structures for the energy and Lorentz factor (e.g. Rossi et al. 2002; Zhang \& Mészáros 2002; Kumar \& Granot 2003):

$$
\frac{\epsilon}{\epsilon_{\mathrm{c}}}=e^{-\theta^{2} / 2 \theta_{\mathrm{c}}^{2}}, \quad \frac{\Gamma_{0}(\theta)-1}{\Gamma_{\mathrm{c}, 0}-1}=e^{-\theta^{2} / 2 \theta_{\mathrm{c}}^{2}},
$$



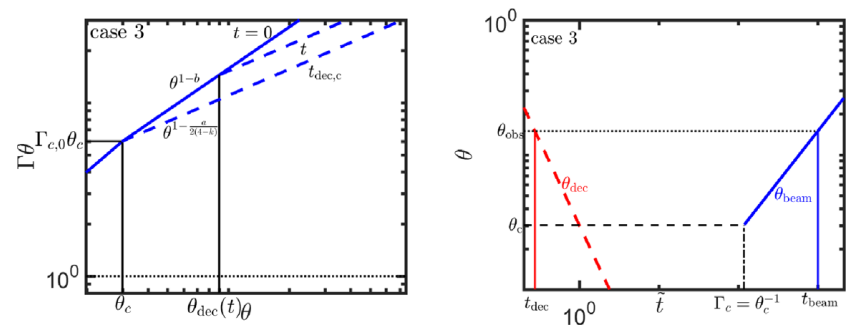

Figure 10. Left: temporal evolution of $\Gamma \theta$ in case $3\left(b<b_{\mathrm{a}}<b_{\mathrm{c}}\right)$. Results are plotted for $\theta_{\mathrm{c}}=0.03, \Gamma_{\mathrm{c}}=200$ as well as $k=0, a=4$, and $b=0.2$. Right: corresponding evolution of the characteristic angles with time. $t_{\mathrm{dec}}<$ $t_{\text {beam }}$ for any observation angle.

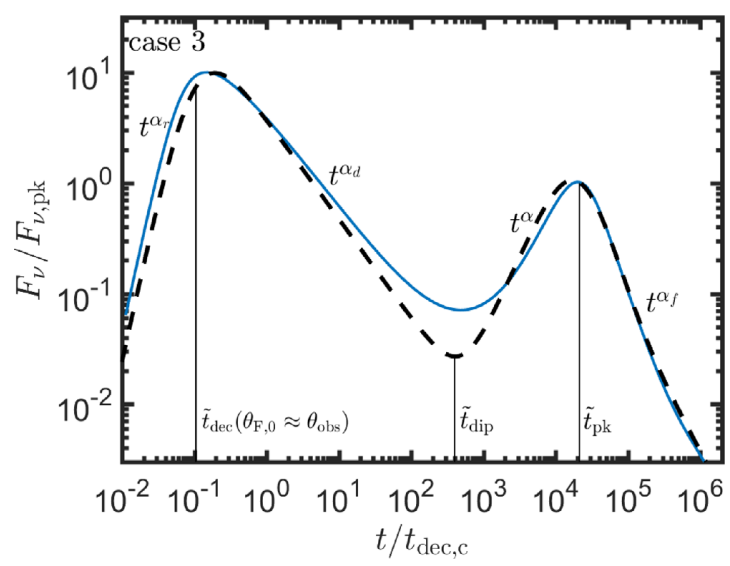

Figure 11. Analytic light curve obtained for case 3 (dashed) as compared with the numerical model of GG18 (solid). Results are shown for $k=0, p=$ 2.2, $a=4, b=0.2, \Gamma_{\mathrm{c}}=100, \theta_{\mathrm{c}}=0.03, \theta_{\mathrm{obs}}=0.5$, and PLS G.
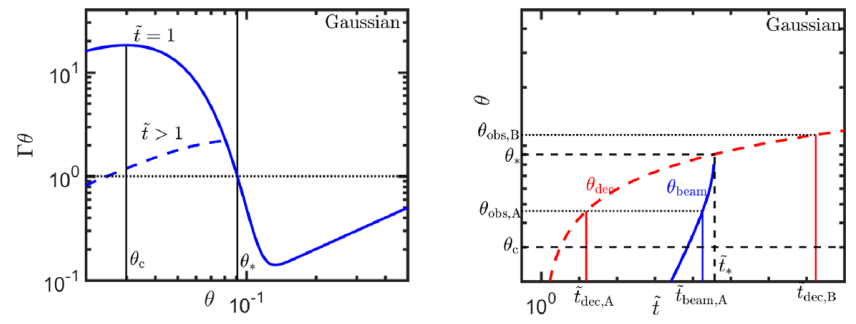

Figure 12. Left: temporal evolution of $\Gamma \theta$ for a Gaussian structured jet. Results are plotted for $\theta_{\mathrm{c}}=0.03, \Gamma_{\mathrm{c}}=1000$ as well as $k=0$. Right: corresponding evolution of the characteristic angles with time.

Following the same derivation outlined in Section 2, one obtains

$\tilde{t}_{\mathrm{dec}}(\theta)=e^{\frac{\theta^{2}}{2 \theta_{\mathrm{c}}^{2}} \frac{7-2 k}{3-k}}$

For $k=0, \tilde{t}_{\mathrm{dec}}(\theta)$ increases with $\theta$. In addition, since $\Gamma(\theta)$ decreases quickly, $\theta_{*}$, defined by the implicit equation

$\xi_{\mathrm{c}} q_{*}^{2} e^{-q_{*}^{2}}=1, \quad q_{*}=\theta_{*} / \theta_{\mathrm{c}}$,

typically satisfies $\theta_{*}<1$. The result is that the Gaussian case is qualitatively similar to case 1 (Section 4.1), with equivalent $\mathrm{A}$ and $\mathrm{B}$ subcases. Namely, if $\theta_{\mathrm{obs}}<\theta_{*}$, then $t_{\mathrm{dec}}\left(\theta_{\mathrm{obs}}\right)<t_{\text {beam }}\left(\theta_{\mathrm{obs}}\right)$, resulting in a double-peaked light curve, and if $\theta_{\mathrm{obs}}>\theta_{*}$, then $t_{\mathrm{dec}}\left(\theta_{\mathrm{obs}}\right)>$ $t_{\text {beam }}\left(\theta_{\text {obs }}\right)$, resulting in a single-peaked light curve. These results are depicted in Figs 12 and 13. Note that a large $\xi_{\mathrm{c}}$ is required in order to have $\theta_{*} \gg \theta_{\mathrm{c}}$ as required in order for the double-peaked light curve to be realized in practice.
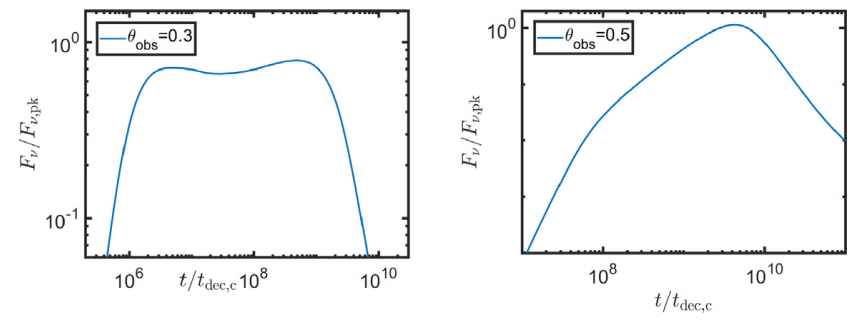

Figure 13. Representative light curves calculated using the numerical model of GG18 applied to Gaussian structured jets. The curves correspond to $\theta_{\mathrm{obs}}<\theta_{*}$ (left) and $\theta_{\mathrm{obs}}>\theta_{*}$ (right). Results are plotted for $\Gamma_{\mathrm{c}, 0}=10^{4}$, $\theta_{\mathrm{c}}=0.08$, and $\theta_{\mathrm{obs}}=0.3,0.5$ respectively.

In the ultrarelativistic limit, the equation for $\theta_{\mathrm{F}, 0}$ is given by

$$
\left(1-\frac{\lambda_{\epsilon}}{\lambda_{\mathcal{D}}}\right)(q-y)^{2}+2 \frac{q-y}{y}=\left(1+\frac{\lambda_{\epsilon}}{\lambda_{\mathcal{D}}}\right) \frac{e^{y^{2}}}{\xi_{\mathrm{c}}}, \quad y \equiv \frac{\theta_{\mathrm{F}, 0}}{\theta_{\mathrm{c}}}
$$

Since closed form algebraic solutions are not available for $\theta_{*}, \theta_{\mathrm{F}, 0}$ in the Gaussian structure case, we plot the numerical solutions for those parameters in Fig. 14.

\section{INFERENCES FROM OBSERVATIONS}

The relationship between the observed characteristic times and fluxes obtained for the different light curves discussed in Section 4 can be used to infer some of the defining physical properties of a GRB.

\subsection{The temporal slopes}

In all the cases considered here, we have found a shallow portion of the light curve, with $F_{v} \propto t^{\alpha}$, that is governed by the temporal evolution of $\theta_{\min }(t)$. The derivation of $\alpha$ for PLS G is given in GG18. Applying the same derivation, we provide here the values also for the other synchrotron PLS in Table 4. In general, relating $\alpha$ to $\beta$ can provide a closure relation between the temporal and spectral slopes of the type $\alpha(\beta, a, k)$ which may be used to test the validity of the model to observations (see e.g. Racusin et al. 2009; Ryan et al. 2019).

In addition, all cases exhibit an early phase, with $F_{v} \propto t^{\alpha_{r}}$, that corresponds to material that has not yet been decelerated and a late decline with $F_{v} \propto t^{\alpha_{f}}$. The value of $k, p$ can be inferred from $\alpha_{\mathrm{r}}, \alpha_{\mathrm{f}}$. For example, for PLS G and $k=0$, assuming no lateral expansion of the jet after the jet-break and as long as the flow is still relativistic, $\alpha_{\mathrm{f}}=-3 p / 4^{5}$ and $\alpha_{\mathrm{r}}=3$ (values for general values of $k$ and other synchrotron PLS are given in Table 4). The value of $p$ can also be extremely well constrained from the spectrum, which is independent of the assumption on lateral expansion. In fact the observations of GRB 170817A spanning all the way from the radio to the X-ray band, and revealing a spectrum consistent with a single PL segment in that frequency range, provided an unprecedented accuracy in determining $p=2.17$ (e.g. D'Avanzo et al. 2018; Lyman et al. 2018; Margutti et al. 2018; Resmi et al. 2018; Troja et al. 2018).

In principle, the value of $a$ can be inferred directly from the asymptotic temporal index $\alpha$ during the rise to the peak that is governed by the angular effect (Gill \& Granot 2018), e.g. for PLS

\footnotetext{
${ }^{5}$ Immediately after the peak, the light curve is slightly steeper due to 'limb-
} brightening' effect (Granot 2007). 

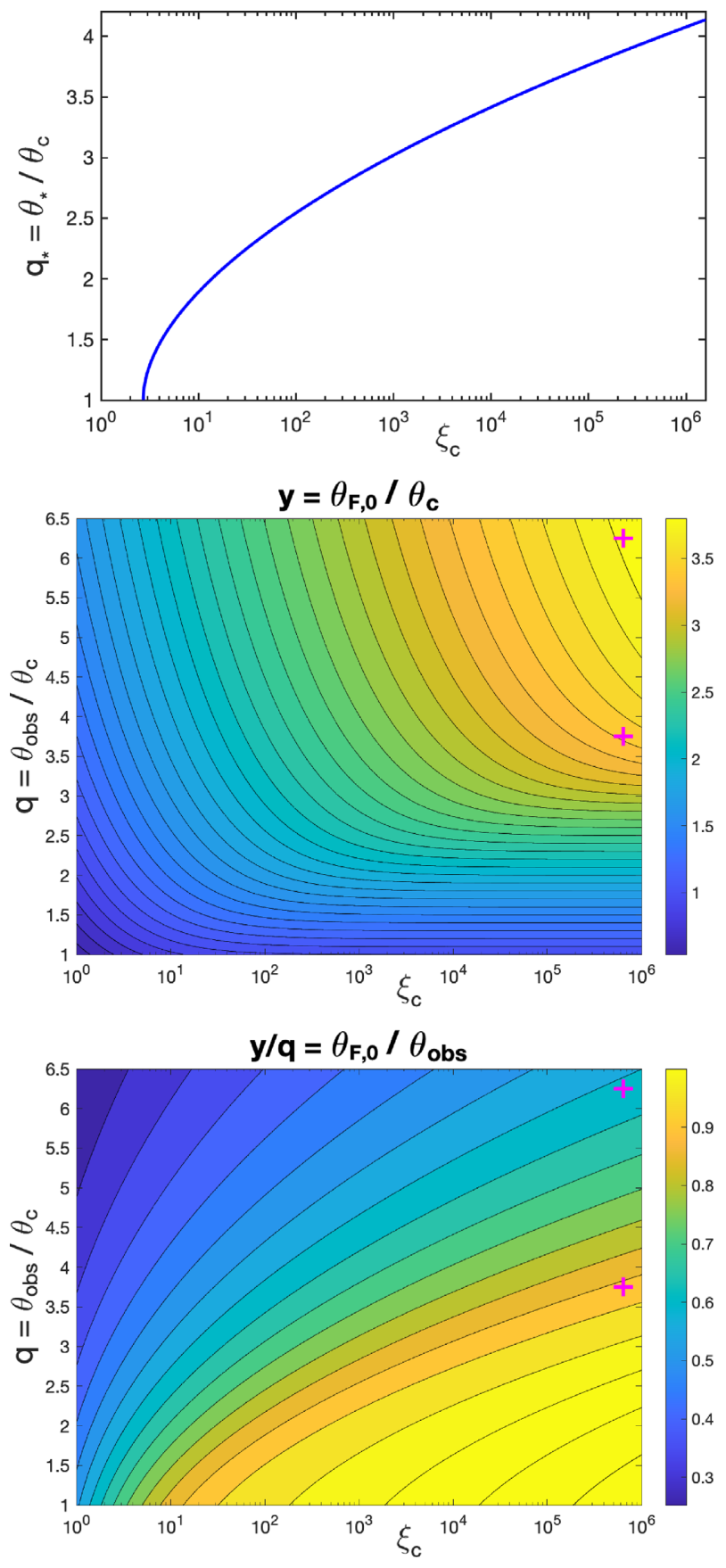

Figure 14. Some key parameters for a Gaussian jet. Top: the angle $\theta_{*}$ defined by $\theta_{*} \Gamma_{0}\left(\theta_{*}\right)=1$ (a solution exists for $\xi_{\mathrm{c}} \geq e \approx 2.718$ ) normalized by $\theta_{\mathrm{c}}$ as a function of $\xi_{\mathrm{c}}=\left(\Gamma_{\mathrm{c}, 0} \theta_{\mathrm{c}}\right)^{2}$ (see equation 28). Middle: contour map of the normalized angle $y=\theta_{\mathrm{F}, 0} / \theta_{\mathrm{c}}$ in the $\xi_{\mathrm{c}}-q$ plane, for PLS G and $p=2.2$ (see equation 29); contours are at intervals of 0.1 from $y=0.5$ to 3.8 , while the two magenta plus symbols in the last two panes are for the two lig htcurves shown in Fig. 13. Bottom: a similar contour map of $y / q=\theta_{\mathrm{F}, 0} / \theta_{\mathrm{obs}}$ with contours at $y / q=0.25: 0.05: 0.95,0.99,0.999,0.9999$, and 0.99999 .

$\mathrm{G}$ (and $k=0$ corresponding to a uniform medium, as relevant for short GRBs),

$a=\frac{8(3-k)}{4(3-k)-4 \alpha-k(p+1)} \stackrel{k \rightarrow 0}{\longrightarrow} \frac{6}{3-\alpha}$.

However, in practice the dynamical range is limited and we are rarely deep in the limit $\theta_{\mathrm{obs}} \gg \theta_{\text {min }} \gg \theta_{\mathrm{c}}$ for which this analytic result holds. For example, in GRB 170817A/GW 170817 the above analytic expression gave $a \approx 2.7$, while a direct fit to the light curve gave $a \approx 4.5$ (Gill \& Granot 2018), since a steeper angular profile is required in order to compensate for the limited dynamical range.

In cases $1 \mathrm{~A}, 2$, and 3, there is another declining phase after the first peak. The asymptotic slope of this decline is similar to that of an on-axis afterglow and depends on the observed synchrotron PLS. We shall denote it by $\alpha_{\mathrm{d}}$ where $F_{v} \propto t^{\alpha_{d}}$. As an example, for PLS $\mathrm{G}$ and $k=0, \alpha_{\mathrm{d}}=-3(p-1) / 4$ (see Table 4 for other cases). We caution the reader that in practice, if the dynamical range between the first peak and the dip is not sufficiently large, the observed decline may be significantly flatter.

\subsection{Time-scales}

All the critical time-scales in determining the observed afterglow light curve are proportional to $t_{\mathrm{dec}, \mathrm{c}} \propto\left(\epsilon_{\mathrm{c}} / A\right)^{\frac{1}{3-k}} \Gamma_{\mathrm{c}, 0}^{\frac{2 k-8}{3-k}}$. In particular, there is a degeneracy between $\epsilon_{\mathrm{c}}, A$ which makes it challenging to infer any one of these parameters on its own. However, since they appear in all the time-scales through the same scaling, comparing the ratio of different light-curve characteristic times is particularly useful for robustly inferring physical properties. We discuss these inferences below.

The time of the main peak in the light curve $t_{\mathrm{pk}}$ for off-axis observers $(q>1)$ can be identified with time at which the jet's core becomes visible, i.e. $\theta_{\mathrm{F}}\left(t_{\mathrm{pk}}\right)=0$. Assuming that the core is initially not visible $\left(\theta_{\mathrm{F}, 0}>\theta_{\mathrm{c}}\right)$, this time is approximately the same as when $\theta_{\min } \rightarrow 0$ using the broken PL description of $\Gamma(t)$

$\tilde{t}_{\mathrm{pk}}=2^{-a /[2(3-k)]} \xi_{\mathrm{c}}^{\frac{4-k}{3-k}} q^{2(4-k) /(3-k)}$.

In cases $1 \mathrm{~A}, 2$, and 3, the light curve is double-peaked. In these cases, two other critical time-scales appear, the time of the first peak, and the time of the dip between the two peaks. These can be estimated in the following way

$$
\begin{aligned}
& \tilde{t}_{\mathrm{pk}}=\tilde{t}_{\mathrm{dec}}\left(\theta_{F, 0}\right) \approx \tilde{t}_{\mathrm{dec}}\left(\theta_{\mathrm{obs}}\right)=\left(1+q^{2}\right)^{[2(4-k) b-a] / 2(3-k)} \\
& \tilde{t}_{\mathrm{dip}} \approx \tilde{t}_{\mathrm{beam}}\left(\theta_{\mathrm{obs}}\right)=2^{\frac{-a}{2(3-k)}}\left(1+q^{2}\right)^{\frac{-a}{2(3-k)}} q^{\frac{2(4-k)}{3-k}} \xi_{\mathrm{c}}^{\frac{4-k}{3-k}}
\end{aligned}
$$

where in the right-hand side of equation (33), we have used a slightly more accurate definition of $t_{\text {dip }}$, namely that the extrapolations of the flux from the first and second peak matches, i.e. $F_{1 \mathrm{p}}\left(t_{\mathrm{dip}} / t_{1 \mathrm{pk}}\right)^{\alpha_{d}}=$ $F_{\mathrm{pk}}\left(t_{\mathrm{dip}} / t_{\mathrm{pk}}\right)^{\alpha}$ (the expressions for those fluxes are given in the next subsection). We note that the difference between $\tilde{t}_{\text {beam }}\left(\theta_{\text {obs }}\right)$ and the more accurate prescription are rather small, up to tens of percent throughout the majority of the parameter space.

Assuming $k, a$ can be determined from the temporal slopes (see above) and at the limit $q \gg 1$ we find

$q=\frac{\theta_{\mathrm{obs}}}{\theta_{\mathrm{c}}} \approx\left(\frac{t_{\mathrm{pk}}}{t_{\mathrm{dip}}}\right)^{\frac{3-k}{a}}$

and

$\xi_{\mathrm{c}} \approx 2^{\frac{a}{2(4-k)}}\left(\frac{t_{\mathrm{pk}}}{t_{1 \mathrm{pk}}}\right)^{\frac{3-k}{4-k}} q^{2(b-1)-\frac{a}{4-k}}$

In case $1 \mathrm{~B}$, the time of the peak remains the same as in equation (31), but the times of the first peak and the dip are no longer relevant. Instead, a new time-scale appears, which is the time at which the shallow rise $\left(F_{v} \propto t^{\alpha}\right)$ starts, $t_{\mathrm{dec}}\left(\theta_{\mathrm{F}, 0}\right)$. In this regime, $\theta_{\mathrm{F}, 0}$ can be well estimated by equation (13). A slightly less accurate but easier approximation for the time of the initial rise is given by $t_{\mathrm{dec}}\left(\theta_{\min , 0}\right)$. When $\theta_{*}<\theta_{\mathrm{obs}}$, the latter is roughly given by 
$\theta_{\min , 0} \approx \theta_{\mathrm{c}} \xi_{\mathrm{c}}^{\frac{1}{2 b}}\left(q-\xi_{\mathrm{c}}^{\frac{1}{2 b}} q^{\frac{1}{b}}\right)^{\frac{1}{b}} \Longrightarrow$

$t_{\mathrm{dec}}\left(\theta_{\min , 0}\right)=\left[1+\xi_{\mathrm{c}}^{\frac{1}{b}}\left(q-\xi_{\mathrm{c}}^{\frac{1}{2 b}} q^{\frac{1}{b}}\right)^{\frac{2}{b}}\right]^{\frac{2(4-k) b-a}{2(3-k)}}$.

Finally, the most straightforward but least accurate expression for the initial rise is given by $\tilde{t}_{\mathrm{dec}}\left(\theta_{\min , 0}\right) \approx \tilde{t}_{*}\left(\theta_{\mathrm{obs}} / \theta_{*}\right)^{[2(4-k) b-a] /(3-k) b}$ (see Section 4) or equivalently

$\tilde{t}_{\mathrm{dec}}\left(\theta_{\min , 0}\right) \approx\left(\xi_{\mathrm{c}} q^{2}\right)^{[2(4-k) b-a] /[2 b(3-k)]}$

In this case, we obtain the following relation between the observables and the physical parameters

$\xi_{\mathrm{c}} q^{2}=\left(\frac{t_{\mathrm{pk}}}{t_{\mathrm{dec}}\left(\theta_{\min , 0}\right)}\right)^{\frac{2 b(3-k)}{a}} 2^{b}$

\subsection{Fluxes}

In cases $1 \mathrm{~A}, 2$, and 3 , there are three characteristic fluxes. For concreteness, we assume that the observed band is in PLS G. The flux at $t_{\mathrm{pk}}$ has been well studied in the literature (e.g. Nakar, Piran \& Granot 2002). For $k=0$, the result is

$F_{\mathrm{pk}} \propto \epsilon_{\mathrm{c}} \theta_{\mathrm{c}}^{2} n^{\frac{p+1}{4}} \epsilon_{\mathrm{e}}^{p-1} \epsilon_{\mathrm{B}}^{\frac{p+1}{4}} v^{\frac{1-p}{2}} d_{\mathrm{L}}^{-2} \theta_{\mathrm{obs}}^{-2 p}$

where $n$ is the particle number density of the circumburst medium, $d_{\mathrm{L}}$ is the luminosity distance of the GRB, and $\epsilon_{\mathrm{e}}$ and $\epsilon_{\mathrm{B}}$ are the shock microphysical parameters representing the fractions of the total internal energy density behind the shock deposited in relativistic electrons and magnetic fields, respectively. Since the peak flux is degenerate between several of the bursts' properties, we consider, as for the time-scales, the fluxes relative to the peak flux. The flux at the first peak can be approximated by noticing that the time and flux of the later peak are directly related to the time and flux at the moment of the jet break (i.e. when $\left.\Gamma\left(\theta=0, t_{\mathrm{j}, \mathrm{b}}\right) \theta_{\mathrm{c}}=1\right)$. Assuming no lateral expansion, the appropriate expressions are $t_{\mathrm{pk}} / t_{\mathrm{j}, \mathrm{b}} \approx\left(\theta_{\mathrm{obs}} / \theta_{\mathrm{c}}\right)^{8 / 3}$, $F_{\mathrm{pk}} / F_{\mathrm{j}, \mathrm{b}} \approx\left(\theta_{\mathrm{obs}} / \theta_{\mathrm{c}}\right)^{-2 p}$ (Nakar et al. 2002). The time and flux at $t_{\mathrm{j}, \mathrm{b}}$ can then be extrapolated back to $t_{1 \mathrm{pk}}$, using the standard spherically symmetric pre-deceleration description of the flux (e.g. Granot \& Sari 2002) and using $E_{\text {iso }} \sim 4 \pi \epsilon(\theta)$. The result is

$F_{1 \mathrm{pk}} \approx F_{\mathrm{pk}}\left(\frac{\theta_{\mathrm{obs}}}{\theta_{\mathrm{c}}}\right)^{\frac{8-a(3+p)}{4}}\left(\frac{t_{1 \mathrm{pk}}}{t_{\mathrm{pk}}}\right)^{\frac{3(1-p)}{4}} \approx 2^{-\frac{a}{8}} \xi_{\mathrm{c}} q^{4-a-2 b}$

where in the right-hand side we have plugged the asymptotic expressions for the time-scales and $p \approx 2$ for clarity. This demonstrates that it is possible for the first peak to be brighter than the second one for large enough $q$ and for sufficiently small $a, b$. Out of the five physical quantities $F_{\mathrm{pk}}, F_{1 \mathrm{pk}}, t_{\mathrm{pk}}, t_{\mathrm{dip}}, t_{1 \mathrm{pk}}$ only four are truly independent (see discussion in the previous subsection regarding $t_{\text {dip }}$ ). There is therefore a choice of which quantities to use, depending on how well they can be determined and what physical quantity is attempted at being deduced.

The flux at $t_{\mathrm{dip}}$ is given by a direct extrapolation from $t_{\mathrm{pk}}$,

$F_{\text {dip }}=F_{\mathrm{pk}}\left(\frac{t_{\mathrm{dip}}}{t_{\mathrm{pk}}}\right)^{\alpha}$

Clearly, $F_{\mathrm{dip}}$ does not provide independent information to that obtained from $t_{\mathrm{dip}} / t_{\mathrm{pk}}$ and the observed temporal slope. The ratio $F_{1 \mathrm{pk}} / F_{\mathrm{pk}}$ is however more illuminating and provides an independent

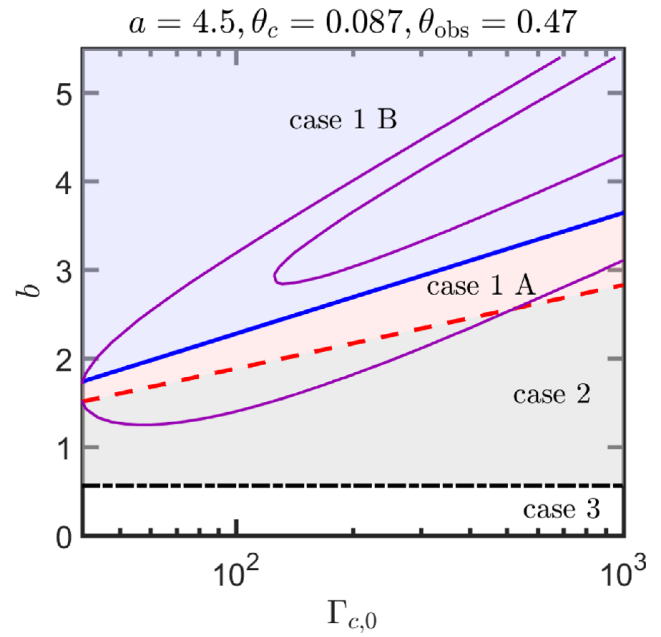

Figure 15. Allowed parameter space for the different types of light curves presented in this paper. The blue solid line depicts $\theta_{\mathrm{obs}}=\theta_{*}$, the red dashed line depicts $b=b_{\mathrm{c}}$, and the dotted-dashed black line depicts $b=b_{\mathrm{a}}$. Results are plotted with $\theta_{\mathrm{c}}=0.087, \theta_{\mathrm{obs}}=0.47$ as inferred for GRB 170817A (as well as $a=4.5$ for the purpose of distinguishing between cases 2 and 3 , the results depend very weakly on the specific value). Light curves with a single peak (case 1B) require large $b$ and / or small $\Gamma_{\mathrm{c}}$ (above the blue line). As the distance from the blue line increases the early peak emerges and becomes gradually stronger. Overplotted in purple are the same $\chi_{v}^{2} \leq 3.2$ and $\leq 2.7$ model fitting contours shown in Fig. 17.

estimate of the observation angle

$q=\left(\frac{F_{1 \mathrm{pk}}}{F_{\mathrm{pk}}}\right)^{\frac{4}{8-a(3+p)}}\left(\frac{t_{\mathrm{pk}}}{t_{1 \mathrm{pk}}}\right)^{\frac{3(1-p)}{8-a(3+p)}}$

In case $1 \mathrm{~B}$, one can express the flux at $t_{\mathrm{dec}}\left(\theta_{\mathrm{F}, 0}\right)$ as

$F\left(t_{\mathrm{dec}}\left(\theta_{\mathrm{F}, 0}\right)\right)=F_{\mathrm{pk}}\left(\frac{t_{\mathrm{dec}}\left(\theta_{\mathrm{F}, 0}\right)}{t_{\mathrm{pk}}}\right)^{\alpha}$.

As in the case of $F_{\text {dip }}$, this does not provide additional information to that given by the equations for the temporal slope and for $t_{\mathrm{dec}}\left(\theta_{\mathrm{F}, 0}\right)$.

\section{GRB 170817A AS A TEST CASE}

We have found in this work four light-curve regimes with two main qualitative types of GRB afterglows, with and without a double peak. The qualitative difference between these regimes has some straightforward implications on the physical parameters.

Consider for example, a situation in which one excludes with confidence the existence of a double peak in a given GRB afterglow. Indeed, this may be the case for GRB 170817A in which the first detections occurred at $\sim 10 \mathrm{~d}$ after the burst, while early observations yielded a strong upper limit on the flux starting from $\sim 1 \mathrm{~d}$ after the trigger. What can be learned from this observation? In order to avoid a first peak, the conditions must be close to those corresponding to case $1 \mathrm{~b}$. Namely (i) $b>b_{\mathrm{c}}\left(\Gamma_{\mathrm{c}, 0}, \theta_{\mathrm{c}}\right)$ and (ii) $\theta_{\mathrm{obs}}>\theta_{*}\left(\Gamma_{\mathrm{c}, 0}\right.$, $\theta_{\mathrm{c}}$ ). Since the values of $\theta_{\mathrm{c}} \approx 0.087, \theta_{\mathrm{obs}} \approx 0.47$ are relatively well constrained from the combination of the superluminal motion observation and the time of the observed peak (Mooley et al. 2018; Pooley et al. 2018; Gill et al. 2019) and since $a$ can be reasonably well constrained from the shallow rise of the light curve towards the peak (see Section 5.1), it is useful to describe the parameter space corresponding to the different light-curve regimes in terms of $b, \Gamma_{\mathrm{c}, 0}$. The results are shown in Fig. 15. Large $b$ and / or small $\Gamma_{\mathrm{c}, 0}$ are needed to completely avoid the first peak. In general, as 


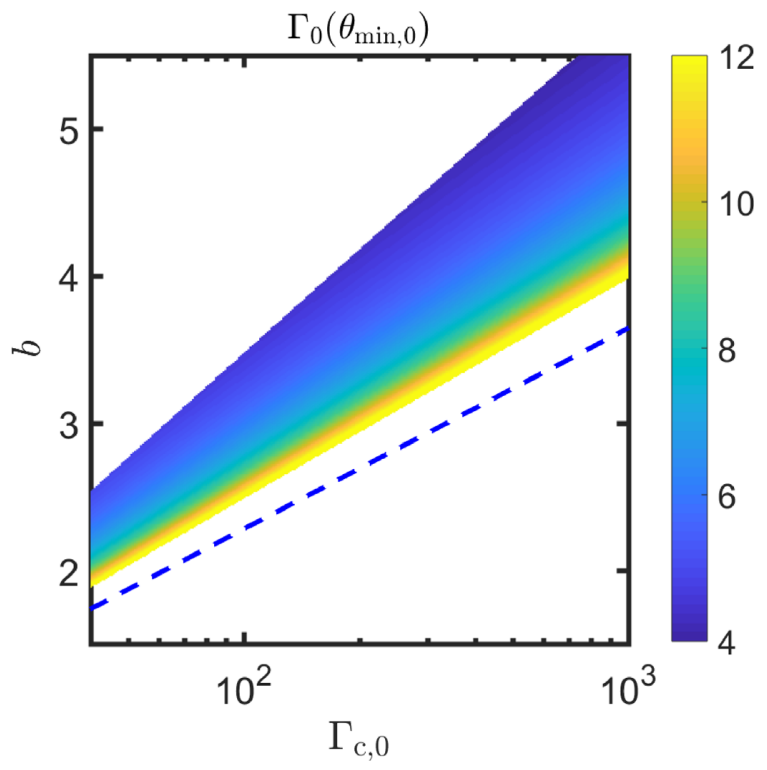

Figure 16. Allowed parameter space provided by the requirements that only one peak is seen in the light curve with $t_{\mathrm{pk}}>7 t_{\mathrm{dec}}\left(\theta_{\min , 0}\right)$ and assuming $a=4.5, \theta_{\mathrm{c}}=0.087$, and $\theta_{\mathrm{obs}}=0.47$. Colour represents the value of $\Gamma_{0}\left(\theta_{\min , 0}\right)$ which in this regime is approximately the initial Lorentz factor of the material dominating the early light curve. A dashed blue line depicts the boundary between cases $1 \mathrm{~B}$ and $1 \mathrm{~A}$ (see Fig. 15).

$b$ becomes smaller, and / or $\Gamma_{\mathrm{c}, 0}$ becomes larger a second peak emerges and gradually becomes stronger.

In GRB 170817A, the peak of the light curve occurred at $t_{\mathrm{pk}} \approx$ $150 \mathrm{~d}$, while the beginning of the shallow rise started at $\approx 10-20 \mathrm{~d}$. The large span of time between the beginning of the shallow rise and the eventual peak, provides another constraint on the allowed parameter space. Assuming no lateral expansion and conservatively taking $t_{\mathrm{pk}} / t_{\mathrm{dec}}\left(\theta_{\min , 0}\right)>7$, we may use equation (39) to further constrain the allowed parameter space. The results are shown in Fig. 16.

In Fig. 17, we show the reduced chi-square $\left(\chi_{v}^{2}\right)$ contour map (top panel) in the plane of $\left\{\Gamma_{\mathrm{c}, 0}, b\right\}$ as obtained from a PL structured jet model fit of GG18, with $a=4.5, \theta_{\mathrm{c}}=0.087, \theta_{\mathrm{obs}}=0.47$, to the afterglow data of GRB 170817A. In the bottom panel, we show the best-fitting light curve from GG18 and Gill et al. (2019) along with shaded regions that encompass light curves obtained for different values of $\left\{\Gamma_{\mathrm{c}, 0}, b\right\}$ that correspond to $\chi_{v}^{2} \leq 3.2$ and $v \leq 2.7$. The parameter space providing the best fits for these models agrees well with the space given by the requirement of having one peak with $t_{\mathrm{pk}}$ $>7 t_{\mathrm{dec}}\left(\theta_{\min , 0}\right)$. Both calculations lead to a narrow allowed region in the $\left\{\Gamma_{\mathrm{c}, 0}, b\right\}$ parameter space, which represents a constraint on $\Gamma_{0}\left(\theta_{\min , 0}\right)$. If the latter is too large then there will be an early peak that quickly becomes too bright compared to the available limits. Instead, if the Lorentz factor of the material dominating the early light curve is too small, then this material takes too long to decelerate and the shallow rise does not last for long enough. The value required by the conditions outlined above leads to $\Gamma_{0}\left(\theta_{\min , 0}\right)$ $\approx 5-7$. From the $\chi_{v}^{2}$ map (regions within the red contours in the top panel) and the corresponding shaded red regions in the light-curve plot, it is clear that PL structured jet models with $b \lesssim 1.2$ and $\Gamma_{\mathrm{c}, 0} \lesssim$ 40 would not fit the afterglow data of GRB 1701817A, particularly at early times at $t<40 \mathrm{~d}$.

The value of $\Gamma_{0}\left(\theta_{\min , 0}\right)$ can be independently understood from the observations of superluminal motion in GRB 170817A (Mooley
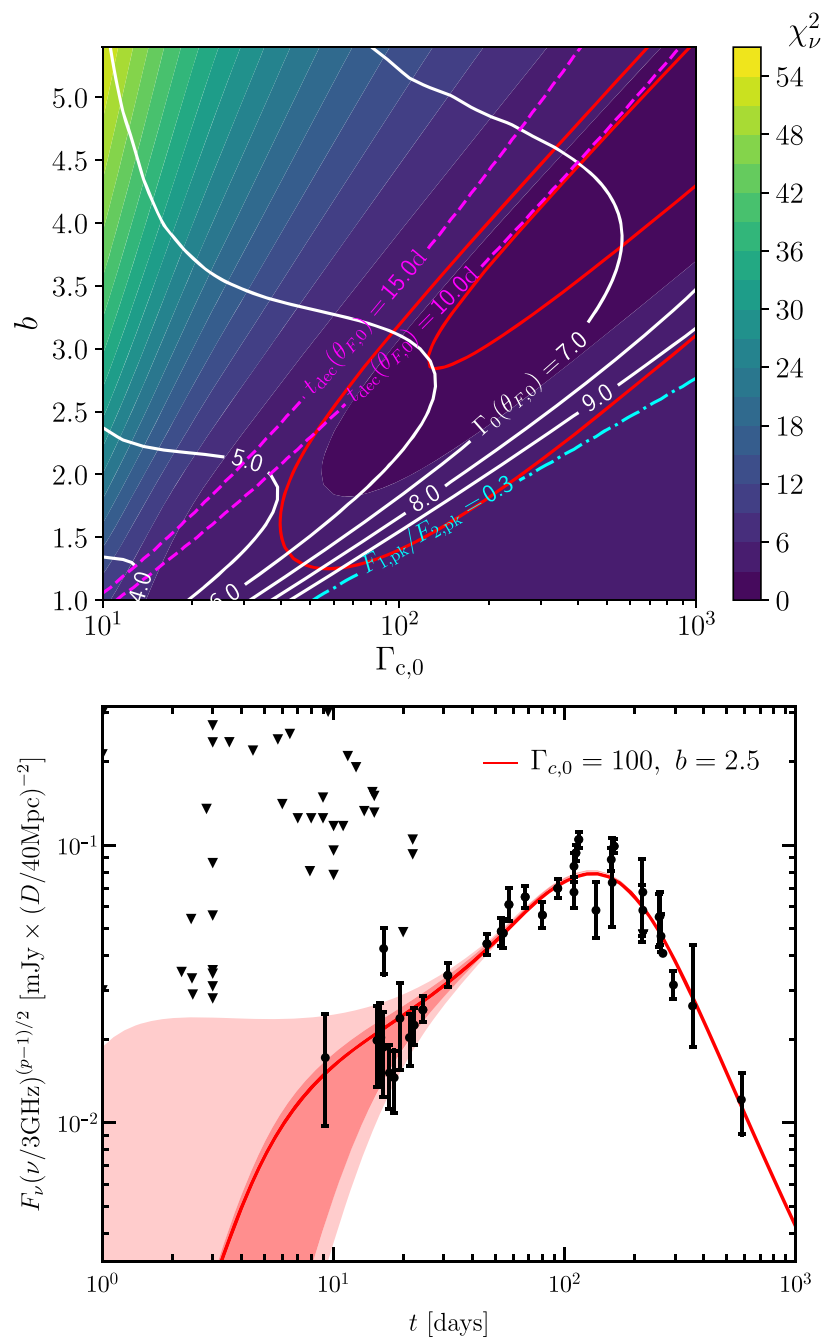

Figure 17. Top: reduced chi-square $\left(\chi_{v}^{2}\right)$ contour map obtained by fitting the PL model of GG18 (with the same $a, \theta_{\mathrm{c}}, \theta_{\mathrm{obs}}$ as in Fig. 15) to the afterglow data of GRB 170817A. The outer and inner solid red curves encompass regions in the parameter space that give the best-fitting solution with $\chi_{v}^{2} \leq 3.2$ and $\leq 2.7$, respectively. These regions are constrained from above by dashed magenta lines for which the parameter space below the lines always yield the condition that $t_{\mathrm{dec}}\left(\theta_{\mathrm{F}, 0}\right)<t_{1}$, where $t_{1}$ are representative times of the early afterglow observations. Another constraint is shown by a dotted-dashed cyan line for which the region above the line always yield the flux ratio $F_{1, \mathrm{pk}} / F_{2, \mathrm{pk}}<0.3$. Solid white contour lines indicate the initial bulk Lorentz factor of the initial angle that dominated the flux. Bottom: PL jet model light-curve fit to the afterglow data of GRB 170817A, with upper limits marked with downward triangles. The solid red curve shows the best-fitting solution of Gill \& Granot (2018) and Gill et al. (2019). The lighter and darker shaded red regions encompass light curves obtained for $\left\{\Gamma_{\mathrm{c}, 0}, b\right\}$ values with best-fitting $\chi_{v}^{2} \leq 3.2$ and $\leq 2.7$, respectively.

et al. 2018; Ghirlanda et al. 2019). These observations have revealed that the flux centroid of GRB 170817A was moving with an apparent velocity of $\approx 4 c$ around the time of the light-curve peak. The implication is that $\Gamma\left(\theta_{\mathrm{c}}, t_{\mathrm{pk}}\right) \approx 4$. This result can be related to the Lorentz factor along the direction initially dominating the light curve, by using the definition of $\theta_{\min }$ (equation 8), $\Gamma_{0}\left(\theta_{\min , 0}\right)=$ $f \Gamma\left(\theta_{\mathrm{c}}, t_{\mathrm{pk}}\right)$, where $f=\left(\theta_{\mathrm{obs}}-\theta_{\mathrm{c}}\right) /\left(\theta_{\mathrm{obs}}-\theta_{\min , 0}\right)$. Since by definition $f \geq 1$, this immediately suggests that $\Gamma_{0}\left(\theta_{\min , 0}\right) \gtrsim 4$. In regime $1 \mathrm{~B}, \theta_{\min , 0} \ll \theta_{\text {obs }}$, implying $f \approx 1$. Using equation (7), we can obtain a rough estimate for $f$ using the ratio $t_{\mathrm{pk}} / t_{\mathrm{dec}}\left(\theta_{\min , 0}\right) \approx$ 
$\left(\theta_{\min , 0} / \theta_{\mathrm{c}}\right)^{a /(3-k)}$. The condition $t_{\mathrm{pk}} / t_{\mathrm{dec}}\left(\theta_{\min , 0}\right)>7$ then implies $\left(\theta_{\min , 0} / \theta_{\mathrm{c}}\right) \gtrsim 3.7$. In reality, as shown in Fig. 1 , the decay of $\theta_{\min }(t)$ is less steep than the asymptotic PL decay. Taking, for example, $\theta_{\text {obs }}, \theta_{\mathrm{c}}, a$ as above, as well as $\Gamma_{\mathrm{c}, 0}=300, b=4$ (where the condition $t_{\mathrm{pk}} / t_{\mathrm{dec}}\left(\theta_{\min , 0}\right)>7$ is satisfied, as shown in Fig. 16) we find $\left(\theta_{\min , 0} / \theta_{\mathrm{c}}\right) \approx 2.8$. Plugging this back into $f$ (and using our values for $\theta_{\mathrm{obs}} / \theta_{\mathrm{c}}$ ) we find $f \approx 1.7$. Overall, we conclude that $\Gamma_{0}\left(\theta_{\min , 0}\right) \approx 7$, which is consistent with what we have found above from the more detailed calculation. Finally, the value of $\Gamma_{0}\left(\theta_{\min , 0}\right)$ is also consistent with the limits for the material dominating the prompt material which from compactness arguments leads to $\Gamma \gtrsim$ 2-3 (e.g. Kasliwal et al. 2017; Matsumoto, Nakar \& Piran 2019a). Summarizing, we find three independent constraints on $\Gamma_{0}\left(\theta_{\min , 0}\right)$ (from the light-curve analysis, from superluminal motion and from compactness limits) which are all in broad agreement with each other. This lends credence to the angular jet structure models considered in this work. Furthermore, it outlines easily applicable consistency checks that can be used for future events to compare between the different models.

\section{CONCLUSIONS}

We studied analytically the shapes of GRB afterglows that arise from structured jets viewed off-axis. We found qualitatively different types of light curves that may be viewed, depending on the jet properties and on the LOS to the observer. Most notably, the light curve may be either singly or doubly peaked, depending on whether $\theta_{\text {obs }}$ is (correspondingly) larger or smaller than a critical angle, $\theta_{*}{ }^{6}{ }^{6}$

GRB afterglow fitting involves many unknown model parameters that quantify the jet properties, the surrounding medium, the shock microphysics, and the observer's viewing angle. Many of these different properties or model parameters are degenerate and cannot be uniquely determined or constrained, even with a very good set of observations, such as e.g. in GRB 170817A/GW 170817. However, focusing on the shape of the light curves, rather than the absolute normalizations of the flux and/or time-scales, immediately removes the dependence on many of those parameters, and can provide very significant constraints on a subset of them.

In the single peak scenario (case 1B), the shape of the light curve provides four scale-free observables: the early rise slope, $\alpha_{\mathrm{r}}$, the shallow rise slope, $\alpha$, the final decline slope, $\alpha_{\mathrm{f}}$, and the ratio between the start and end of the shallow rise phase, $t_{\mathrm{dec}}\left(\theta_{\min , 0}\right) / t_{\mathrm{pk}}$. The first three can constrain $k, p$, and $a .^{7}$ The fourth condition then provides a specific relation between $\xi_{\mathrm{c}}, b$, and $q$. If one has some additional knowledge of $\theta_{\mathrm{c}}, \theta_{\mathrm{obs}}$, for example from superluminal motion observations and/or from the width of the light-curve peak, then the $t_{\mathrm{dec}}\left(\theta_{\min , 0}\right) / t_{\mathrm{pk}}$ constraint can be reduced to a simple relation between $b$ and $\Gamma_{\mathrm{c}, 0}$. This leads to an estimate of the initial Lorentz factor along the LOS that can be independently tested by superluminal motion observations and/or compactness of the prompt emission. Applying this analysis to GRB 170817A, we find the Lorentz factor of material moving along the LOS to the

\footnotetext{
${ }^{6}$ When $\theta_{*}$ becomes ill-defined or $\theta_{*} \gtrsim 1$, the light curve is always doubly peaked, see Section 4.

${ }^{7}$ In reality it may be challenging to observe the early rise slope. In X-rays, it may be overshadowed by 'internal' emission (i.e. from below the forward shock) associated with central engine activity (Lü et al. 2015; Beniamini \& Mochkovitch 2017). In the optical, it may be overshadowed by the kilonova emission (Kasen et al. 2017). Finally, in the radio it may be overshadowed by reverse shock emission (Lamb \& Kobayashi 2019).
}

observer to be $\Gamma_{0}\left(\theta_{\min , 0}\right)=5-7$ as well as $b \gtrsim 1.2, \Gamma_{\mathrm{c}, 0} \gtrsim 40$. These are the first direct limits from a single event viewed offaxis, which show that the core must have been ultrarelativistic, with a much larger Lorentz factor than the material that dominated the observed emission. This has implications, for example, for the prompt emission phase of GRBs, in which, depending on the radiation mechanism, the Lorentz factor may have a profound effect on the $\gamma$-ray spectrum and light curve. For example, Beniamini \& Nakar (2019) have recently studied structured jet models and have shown that, at least in long GRBs, the efficiency of $\gamma$-ray production must be significantly diminished for $\Gamma_{0}\left(\theta_{\min , 0}\right) \lesssim 50$ (see also Hascoët et al. 2014; Ghirlanda et al. 2018; Matsumoto, Nakar \& Piran 2019b), effectively shutting off the prompt emission far beyond the jet's core.

In the double peak scenario, the shape of the light curve depends on five independent scale-free observables. These can be chosen in different ways. One useful such set is the four temporal slopes: $\alpha_{\mathrm{r}}$, $\alpha, \alpha_{\mathrm{d}}, \alpha_{\mathrm{f}}$, and the ratio of the the early and late peak times $t_{1 \mathrm{pk}} / t_{\mathrm{pk}}$. Once more, the slopes constrain $k, p$, and $a$, while the time ratio provides a relation between $\xi_{\mathrm{c}}, b$, and $q$. This relation can be reduced to a relation between $b$ and $\Gamma_{\mathrm{c}, 0}$ in a similar way to that described above for the singly peaked scenario. It is worth noting that reverse shock emission may also lead to an early peak in the afterglow light curve, predominantly in the radio band (Lamb et al. 2019), which is physically distinct from the origin of the early peak described in this work. In case an early peak is detected, multiwavelength observations and/or spectral analysis of the light curve, could potentially be used to distinguish between the different scenarios.

Only a fraction of GW-detected binary neutron star mergers will have a detectable electromagnetic signal (e.g. Lamb \& Kobayashi 2017; Beniamini et al. 2019; Duque, Daigne \& Mochkovitch 2019b; Kathirgamaraju, Giannios \& Beniamini 2019). Current predictions suggest that between the main electromagnetic counterparts: the prompt GRB emission, the kilonova (and/or its afterglow) and the GRB afterglow, it is the latter that is likely to be detected in GW triggered events most often (Duque et al. 2019b). It is therefore of great importance to understand what physical parameters can be directly probed by such detections. Indeed, the detection fraction alone, can be used to statistically constrain the energy of the explosions and the typical densities of the surrounding medium (e.g. Duque et al. 2019a; Beniamini \& Piran 2019). The analysis described in this work can be used to significantly enhance numerical fitting attempts by potentially eliminating large portions of the initial parameter space as well as aiding with providing a physical interpretation and testable predictions for the model.

\section{ACKNOWLEDGEMENTS}

PB's research was funded in part by the Gordon and Betty Moore Foundation through grant GBMF5076. This research was also supported by the Israel Science Foundation (ISF)-National Natural Science Foundation of China (NSFC) joint research program (grant no. 3296/19; RG and JG). PB thanks Wenbin Lu, Geoffrey Ryan, Pawan Kumar, and Ilaria Caiazzo for helpful discussions. We also thank Shiho Kobayashi for useful comments and the anonymous referee for a constructive report.

\section{REFERENCES}

Abbott B. P. et al., 2017a, Phys. Rev. Lett., 119, 161101

Abbott B. P. et al., 2017b, ApJ, 848, L12

Alexander K. D. et al., 2018, ApJ, 863, L18 
Beniamini P., Mochkovitch R., 2017, A\&A, 605, A60

Beniamini P., Nakar E., 2019, MNRAS, 482, 5430

Beniamini P., Piran T., 2019, MNRAS, 487, 4847

Beniamini P., Petropoulou M., Barniol Duran R., Giannios D., 2019, MNRAS, 483, 840

Beniamini P., Barniol Duran R., Petropoulou M., Giannios D., 2020a, preprint (arXiv:2001.00950)

Beniamini P., Duque R., Daigne F., Mochkovitch R., 2020b, MNRAS, 492 , 2847

Blandford R. D., McKee C. F., 1976, Phys. Fluid, 19, 1130

D'Avanzo P. et al., 2018, A\&A, 613, L1

De Colle F., Ramirez-Ruiz E., Granot J., Lopez-Camara D., 2012, ApJ, 751, 57

Duque R., Beniamini P., Daigne F., Mochkovitch R., 2019a, preprint (arXiv: 1911.03302)

Duque R., Daigne F., Mochkovitch R., 2019b, A\&A, 631, A39

Eichler D., Granot J., 2006, ApJ, 641, L5

Ghirlanda G. et al., 2018, A\&A, 609, A112

Ghirlanda G. et al., 2019, Science, 363, 968

Gill R., Granot J., 2018, MNRAS, 478, 4128

Gill R., Granot J., De Colle F., Urrutia G., 2019, ApJ, 883, 15

Gottlieb O., Nakar E., Piran T., Hotokezaka K., 2018, MNRAS, 479, 588

Granot J., 2005, ApJ, 631, 1022

Granot J., 2007, Rev. Mex. Astron. Astrofis., 27, 140

Granot J., Kumar P., 2003, ApJ, 591, 1086

Granot J., Piran T., 2012, MNRAS, 421, 570

Granot J., Sari R., 2002, ApJ, 568, 820

Granot J., Ramirez-Ruiz E., Perna R., 2005, ApJ, 630, 1003

Granot J., Guetta D., Gill R., 2017, ApJ, 850, L24

Granot J., De Colle F., Ramirez-Ruiz E., 2018, MNRAS, 481, 2711

Hajela A. et al., 2019, ApJ, 886, L17

Hascoët R., Beloborodov A. M., Daigne F., Mochkovitch R., 2014, ApJ, 782,5

Kasen D., Metzger B., Barnes J., Quataert E., Ramirez-Ruiz E., 2017, Nature, 551, 80

Kasliwal M. M. et al., 2017, Science, 358, 1559

Kathirgamaraju A., Barniol Duran R., Giannios D., 2018, MNRAS, 473, L121

Kathirgamaraju A., Giannios D., Beniamini P., 2019, MNRAS, 487, 3914
Kumar P., Granot J., 2003, ApJ, 591, 1075

Lamb G. P., Kobayashi S., 2017, MNRAS, 472, 4953

Lamb G. P., Kobayashi S., 2018, MNRAS, 478, 733

Lamb G. P., Kobayashi S., 2019, MNRAS, 489, 1820

Lamb G. P., Mandel I., Resmi L., 2018, MNRAS, 481, 2581

Lamb G. P. et al., 2019, ApJ, 870, L15

Lazzati D., Deich A., Morsony B. J., Workman J. C., 2017, MNRAS, 471, 1652

Lazzati D., Perna R., Morsony B. J., Lopez-Camara D., Cantiello M., Ciolf R., Giacomazzo B., Workman J. C., 2018, Phys. Rev. Lett., 120, 241103

Lü H.-J., Zhang B., Lei W.-H., Li Y., Lasky P. D., 2015, ApJ, 805, 89

Lyman J. D. et al., 2018, Nat. Astron., 2, 751

Margutti R. et al., 2018, ApJ, 856, L18

Matsumoto T., Nakar E., Piran T., 2019a, MNRAS, 483, 1247

Matsumoto T., Nakar E., Piran T., 2019b, MNRAS, 486, 1563

Mooley K. P. et al., 2018, Nature, 561, 355

Nakar E., Piran T., 2018, MNRAS, 478, 407

Nakar E., Piran T., Granot J., 2002, ApJ, 579, 699

Oganesyan G., Ascenzi S., Branchesi M., Sharan Salafia O., Dall'Osso S., Ghirlanda G., 2019, preprint (arXiv:1904.08786)

Panaitescu A., Kumar P., 2000, ApJ, 543, 66

Panaitescu A., Kumar P., 2003, ApJ, 592, 390

Panaitescu A., Mészáros P., 1999, ApJ, 526, 707

Pooley D., Kumar P., Wheeler J. C., Grossan B., 2018, ApJ, 859, L23

Racusin J. L. et al., 2009, ApJ, 698, 43

Resmi L. et al., 2018, ApJ, 867, 57

Rhoads J. E., 1999, ApJ, 525, 737

Rossi E., Lazzati D., Rees M. J., 2002, MNRAS, 332, 945

Rossi E. M., Lazzati D., Salmonson J. D., Ghisellini G., 2004, MNRAS, 354,86

Ryan G., van Eerten H., Piro L., Troja E., 2019, preprint (arXiv:1909.11691)

Sari R., Piran T., Halpern J. P., 1999, ApJ, 519, L17

Takahashi K., Ioka K., 2019, preprint (arXiv:1912.01871)

Troja E. et al., 2018, MNRAS, 478, L18

Xie X., Zrake J., MacFadyen A., 2018, ApJ, 863, 58

Zhang B., Mészáros P., 2002, ApJ, 571, 876

This paper has been typeset from a $\mathrm{T}_{\mathrm{E}} \mathrm{X} / \mathrm{L} \mathrm{T}_{\mathrm{E}} \mathrm{X}$ file prepared by the author. 